ESAIM: COCV 21 (2015) 378-398

DOI: $10.1051 / \mathrm{cocv} / 2014027$
ESAIM: Control, Optimisation and Calculus of Variations

www.esaim-cocv.org

\title{
UNIQUE CONTINUATION FOR STOCHASTIC HEAT EQUATIONS *
}

\author{
QI L $\ddot{U}^{1}$ AND ZHONGQI YIN ${ }^{2}$
}

\begin{abstract}
We establish a unique continuation property for stochastic heat equations evolving in a domain $G \subset \mathbb{R}^{n}(n \in \mathbb{N})$. Our result shows that the value of the solution can be determined uniquely by means of its value on an arbitrary open subdomain of $G$ at any given positive time constant. Further, when $G$ is convex and bounded, we also give a quantitative version of the unique continuation property. As applications, we get an observability estimate for stochastic heat equations, an approximate result and a null controllability result for a backward stochastic heat equation.
\end{abstract}

Mathematics Subject Classification. 60H15, 93B05.

Received October 21, 2013. Revised April 24, 2014.

Published online December 9, 2014.

\section{INTRODUCTION}

We are concerned with the unique continuation for solutions to stochastic heat equations and its application in approximate and null controllability problems for backward stochastic heat equation. Let us consider the following stochastic heat equation:

$$
\mathrm{d} y-\Delta y \mathrm{~d} t=a y \mathrm{~d} t+b y \mathrm{~d} B(t) \quad \text { in } G \times(0, T) .
$$

Here, $T>0$ is arbitrarily given and $G \subset \mathbb{R}^{n}(n \in \mathbb{N})$ is a domain. Let $G_{0} \subset \subset G$ be a subdomain.

We need to introduce some notations to be used in the context. By $\left(\Omega, \mathcal{F},\left\{\mathcal{F}_{t}\right\}_{t \geq 0}, P\right)$, we denote a complete filtered probability space, on which a standard one dimensional Brownian motion $\{B(t)\}_{t \geq 0}$ is defined. Let $H$ be a Fréchet space. We use the symbol $L_{\mathcal{F}}^{2}(0, T ; H)$ to stand for the Fréchet space of all $H$-valued and $\left\{\mathcal{F}_{t}\right\}_{t \geq 0^{-}}$ adapted processes $X(\cdot)$ such that $\mathbb{E}\left(\|X(\cdot)\|_{L^{2}(0, T ; H)}^{2}\right)<\infty$. By the same manner, we adopt the symbols $L_{\mathcal{F}}^{\infty}(0, T ; H)$ to denote the Fréchet space consisting of all $H$-valued $\left\{\mathcal{F}_{t}\right\}_{t \geq 0}$-adapted bounded processes and $L_{\mathcal{F}}^{2}(\Omega ; C([0, T] ; H))$ the Fréchet space consisting of all $H$-valued $\left\{\mathcal{F}_{t}\right\}_{t \geq 0}$-adapted continuous processes $X(\cdot)$ such that $\mathbb{E}\left(\|X(\cdot)\|_{C([0, T] ; H)}^{2}\right)<\infty$. By $L_{\mathcal{F}_{t}}^{2}(\Omega ; H), 0 \leq t \leq T$, we mean the Fréchet space consisting of all $H$-valued $\mathcal{F}_{t}$ measurable variables. All the above spaces are endowed with the canonical quasi-norms.

Keywords and phrases. Stochastic heat equations, unique continuation property, backward stochastic heat equations, approximate controllability, null controllability.

* This work is partially ERC advanced grant 266907 (CPDENL) of the 7th Research Framework Programme (FP7), the NSF of China under grant 11101070, the project MTM2011-29306 of the Spanish Science and Innovation Ministry, the Fundamental Research Funds for the Central Universities in China under grants ZYGX2012J115 and the General Fund Project of Sichuan Provincial Department of Education in China under the grant 124632.

1 School of Mathematics, Sichuan University, Chengdu 610064, P.R. China. Iuqi59@163.com

2 School of Mathematics, Sichuan Normal University, Chengdu 610068, P.R. China. zhongqi.yin@yahoo.com 
We assume that the coefficients of equation (1.1) satisfy the following condition:

$$
a \in L_{\mathcal{F}}^{\infty}\left(0, T ; L_{\mathrm{loc}}^{\infty}(G)\right), \quad b \in L_{\mathcal{F}}^{\infty}\left(0, T ; W_{\mathrm{loc}}^{1, \infty}(G)\right) .
$$

The definition to the solution of equation (1.1) is given as follows.

Definition 1.1. We call $y \in L_{\mathcal{F}}^{2}\left(\Omega ; C\left([0, T] ; L_{\text {loc }}^{2}(G)\right)\right) \cap L_{\mathcal{F}}^{2}\left(0, T ; H_{\text {loc }}^{1}(G)\right)$ a solution to equation (1.1) if for any $t \in[0, T]$, any open set $G^{\prime} \subset \subset G$ and any $\varphi \in H_{0}^{1}\left(G^{\prime}\right)$, it holds that

$$
\begin{aligned}
\int_{G^{\prime}} y(t, x) \varphi(x) \mathrm{d} x-\int_{G^{\prime}} y(0, x) \varphi(x) \mathrm{d} x= & \int_{0}^{t} \int_{G^{\prime}}[-\nabla y(s, x) \cdot \nabla \varphi(x)+a(s, x) y(s, x) \varphi(x)] \mathrm{d} x \mathrm{~d} s \\
& +\int_{0}^{t} \int_{G^{\prime}} b(s, x) y(s, x) \varphi(x) \mathrm{d} x \mathrm{~d} B(s), \quad P \text {-a.s. }
\end{aligned}
$$

We have the following result.

Theorem 1.2. For any given time $T_{0} \in(0, T]$, let $y$ be a solution to equation $(1.1)$ such that $y\left(\cdot, T_{0}\right)=0$ in $G_{0}$, $P$-a.s. Then, $y\left(\cdot, T_{0}\right)=0$ in $G, P$-a.s. Suppose, in addition, that $a \in L_{\mathcal{F}}^{\infty}\left(0, T ; L^{\infty}(G)\right), b \in L_{\mathcal{F}}^{\infty}\left(0, T ; W^{1, \infty}(G)\right)$ and $y=0$ on $\partial G \times(0, T)$, P-a.s. Then $y=0$ in $G \times(0, T), P$-a.s.

Remark 1.3. If $b \in L_{\mathcal{F}}^{\infty}\left(0, T ; W_{\text {loc }}^{2, \infty}(G)\right)$ and $b_{t} \in L_{\mathcal{F}}^{\infty}\left(0, T ; W_{\text {loc }}^{2, \infty}(G)\right)$, one can obtain Theorem 1.2 in a simple way.

Let

$$
z=\mathrm{e}^{\ell} y, \quad \ell=-b(t, x) B(t) .
$$

In light of Itô's formula, it follows that

$$
\begin{aligned}
\mathrm{d} z & =y \mathrm{de}^{\ell}+\mathrm{e}^{\ell} \mathrm{d} y+\mathrm{d} y \mathrm{de}^{\ell} \\
& =-y \mathrm{e}^{\ell}\left[b_{t} B \mathrm{~d} t+b \mathrm{~d} B(t)\right]+\mathrm{e}^{\ell}[\Delta y \mathrm{~d} t+a y \mathrm{~d} t+b y \mathrm{~d} B(t)]-b^{2} \mathrm{e}^{\ell} y \mathrm{~d} t \\
& =\left(-b_{t} B z+\mathrm{e}^{\ell} \Delta y+a z-b^{2} z\right) \mathrm{d} t \\
& =\Delta z \mathrm{~d} t-\left(b_{t} B-a+b^{2}+B \Delta b\right) z \mathrm{~d} t+2 \mathrm{e}^{\ell} B \nabla b \cdot \nabla y \mathrm{~d} t \\
& =\Delta z \mathrm{~d} t-\left(b_{t} B-a+b^{2}+B \Delta b+|\nabla b|^{2} B^{2}\right) z \mathrm{~d} t+2 B \nabla b \cdot \nabla z \mathrm{~d} t .
\end{aligned}
$$

Hence, we know that $z$ solves the following heat equation with random coefficients

$$
z_{t}-\Delta z=2 \mathrm{e}^{\ell} B \nabla b \cdot \nabla z-\left(b_{t} B-a+b^{2}+B \Delta b+|\nabla b|^{2} B^{2}\right) z \quad \text { in } G \times(0, T) .
$$

For a fixed $\omega \in \Omega, z(\cdot, \cdot, \omega)$ is a solution of a heat equation. From the unique continuation property of heat equations (see $[3,22,23]$ for example), we have that $z\left(\cdot, T_{0}, \omega\right)=0$ in $G$, provided that $z\left(\cdot, T_{0}, \omega\right)=0$ in $G_{0}$. On the other hand, since $y\left(\cdot, T_{0}, \cdot\right)=0$ in $G_{0}, P$-a.s., we have $z\left(\cdot, T_{0}, \cdot\right)=0$ in $G_{0}, P$-a.s. Thus, we conclude that $z\left(\cdot, T_{0}, \cdot\right)=0$ in $G, P$-a.s., which leads to that $y\left(\cdot, T_{0}, \cdot\right)=0$ in $G, P$-a.s. Further, if $y=0$ on $\partial G \times(0, T)$, we get $z=0$ on $\partial G \times(0, T)$. This, together with $z\left(\cdot, T_{0}, \cdot\right)=0$ in $G, P$-a.s., implies that $y=z=0$ in $G \times(0, T)$.

Although the strategy given in this remark is simple, it does not work for our purpose in the present paper. Our method has its own interest.

First, we can relax the regularity for the coefficients. This is especially important when one deals with semilinear equations. Let us consider the following example.

$$
\mathrm{d} w-\partial_{x x} w \mathrm{~d} t=w^{m} \mathrm{~d} B(t) \quad \text { in }(0, L) \times(0, T) .
$$

Here $L>0$ and $m \in \mathbb{N}$. From Theorem 1.2, we can conclude that if

$$
w_{1}, w_{2} \in C_{\mathcal{F}}\left([0, T] ; H_{\mathrm{loc}}^{2}(0, L)\right) \cap L_{\mathcal{F}}^{2}\left(\Omega ; C\left([0, T] ; H_{\mathrm{loc}}^{1}(0, L)\right)\right)
$$


solves $(1.4)$ and $w_{1}\left(\cdot, T_{0}\right)=w_{2}\left(\cdot, T_{0}\right)$ in $G_{0}, P$-a.s., then $w_{1}\left(\cdot, T_{0}\right)=w_{2}\left(\cdot, T_{0}\right)$ in $G, P$-a.s. Indeed, let $w_{3}=$ $w_{1}-w_{2}$, then $w_{3}$ solves

$$
\mathrm{d} w_{3}-\partial_{x x} w_{3} \mathrm{~d} t=\tilde{b} w_{3} \mathrm{~d} B(t) \quad \text { in }(0, L) \times(0, T)
$$

where $\tilde{b}=\sum_{j=0}^{m-1} w_{1}^{j} w_{2}^{m-1-j}$. By Sobolev's embedding theorem, $\tilde{b} \in L_{\mathcal{F}}^{\infty}\left(0, T ; W_{\text {loc }}^{1, \infty}(0, L)\right)$. Then, by Theorem $1.2, w_{3}\left(\cdot, T_{0}\right)=0$ in $G, P$-a.s. Clearly, $\tilde{b}$ will never be absolutely continuous in the time variable $t$. Hence, $\tilde{b}_{t} \notin L_{\mathcal{F}}^{\infty}\left(0, T ; W_{\text {loc }}^{2, \infty}(0, L)\right)$.

Second, if $G$ is convex, by our method, we can establish a quantitative version of the unique continuation property. It seems that this cannot be obtained by the strategy introduced above. Indeed, as far as we know, to obtain an inequality like (1.8) for the solution to equation (1.3), we need the coefficients of $\nabla z$ and $z$ to be essentially bounded with respect to $\omega$. However, it is known that the Brownian motion $B$ does not meet this condition.

Further, if we put some more assumptions on $G, a, b$ and $y$, we can get a better result than Theorem 1.2. More precisely, we assume that

Condition 1.4. (1) The domain $G$ is bounded and convex;

$$
\text { (2) } a \in L_{\mathcal{F}}^{\infty}\left(0, T ; L^{\infty}(G)\right), \quad b \in L_{\mathcal{F}}^{\infty}\left(0, T ; W^{1, \infty}(G)\right), \quad y=0 \text { on } \partial G \times(0, T) .
$$

Under Condition 1.4, the solution to equation (1.1) is now given in the following sense.

Definition 1.5. We call $y \in L_{\mathcal{F}}^{2}\left(\Omega ; C\left([0, T] ; L^{2}(G)\right)\right) \cap L_{\mathcal{F}}^{2}\left(0, T ; H_{0}^{1}(G)\right)$ a solution to equation (1.1) if for any $t \in[0, T]$ and any $\varphi \in H_{0}^{1}(G)$, it holds that

$$
\begin{aligned}
\int_{G} y(t, x) \varphi(x) \mathrm{d} x-\int_{G} y(0, x) \varphi(x) \mathrm{d} x= & \int_{0}^{t} \int_{G}[-\nabla y(s, x) \cdot \nabla \varphi(x)+a(s, x) y(s, x) \varphi(x)] \mathrm{d} x \mathrm{~d} s \\
& +\int_{0}^{t} \int_{G} b(s, x) y(s, x) \varphi(x) \mathrm{d} x \mathrm{~d} B(s), \quad P \text {-a.s. }
\end{aligned}
$$

Clearly, the $y$ satisfing Definition 1.5 must satisfy Definition 1.1 .

We have the following result.

Theorem 1.6. Assume that Condition 1.4 holds. For any $T_{0} \in(0, T]$, there exist two constants $C>0$ and $\delta \in(0,1)$ such that for any solution $y$ of $(1.1)$, it holds that

$$
\left\|y\left(T_{0}\right)\right\|_{L^{2}\left(\Omega ; L^{2}(G)\right)} \leq C\|y(0)\|_{L^{2}\left(\Omega ; L^{2}(G)\right)}^{1-\delta}\left\|y\left(T_{0}\right)\right\|_{L^{2}\left(\Omega ; L^{2}\left(G_{0}\right)\right)}^{\delta} .
$$

Remark 1.7. Inequality (1.7) is a quantitative version of the unique continuation property for the solution $y$ with respect to $G_{0} \times\left\{T_{0}\right\}$. Indeed, if $y$ solves equation (1.1) and $y\left(\cdot, T_{0}\right)=0$ in $G_{0}, P$-a.s., then by (1.7) and the backward uniqueness of the stochastic heat equations (see Lem. 2.3 given in the next section), it is clear that $y$ vanishes in $G, P$-a.s.

An obvious drawback of Theorem 1.6 is that we need the convexity of $G$, which is crucial in the proof. How to drop it has its own independent interest.

The research of unique continuation for solutions to partial differential equations originated from the classical Cauchy-Kovalevskaya theorem. It was studied extensively in the literature. We refer the readers to [12,31] and the rich references therein in this respect. Besides its own interest in the partial differential equation theory, it also plays very important roles in both Inverse Problems and Control Theory (see $[13,30]$ for example). The classical unique continuation property is of qualitative nature. It guarantees that the value of the solution in a given domain $\mathcal{M}_{1}$ can be uniquely determined by that of the solution in a suitable subdomain $\mathcal{M}_{2}$ of $\mathcal{M}_{1}$. Once the unique continuation property holds, a natural question is whether one can find a way to recover the 
solution in $\mathcal{M}_{1}$ by the values of the solution in $\mathcal{M}_{2}$. It is well known that the noncharacteristic Cauchy problem is ill-posed, i.e., a small error on the data in $\mathcal{M}_{2}$ may cause uncontrollable effects on the solution in $\mathcal{M}_{1}$ (see [11] for example). Therefore, it is important to have stability estimate for the solution. We refer the readers to [13] for an introduction for this subject. In general, such kind of estimates can be divided into two classes:

1. Observability estimate. The common form reads

$$
\|y\|_{\mathcal{M}_{1}} \leq \mathcal{C}\|y\|_{\mathcal{M}_{2}},
$$

where $\|\cdot\|_{\mathcal{M}_{i}}, i=1,2$ denote some suitable norms for the restriction of the solution $y$ on $\mathcal{M}_{i}, i=1,2$, respectively, and $\mathcal{C}$ is a constant independent of $y$. We refer the readers to $[10,25]$ for the observability estimate for heat equations and stochastic heat equations, respectively.

2. Quantitative version of unique continuation. It also appears in the form of (1.8) but with $\mathcal{C}$ depending on $y$. One can turn to $[3,4,15,20]$ for such kind of estimates for heat equations and its stochastic counterpart, respectively.

Although there are numerous references addressing to unique continuation properties for deterministic heat equations (see $[3,4,16,20,22,23,26]$ for example), very little is known for the stochastic counterpart and it remains to be further understood. As far as we know, $[15,28]$ are the only two publications in this field. The result in reference [28] shows that a solution to the stochastic heat equation (without boundary condition) evolving in $G$ would vanish almost surely, provided that it vanishes in $G_{0} \times(0, T), P$-a.s. In reference [15], the authors proved that a solution to the stochastic heat equation (with a partial homogeneous Dirichlet boundary condition on arbitrary open subset $\Gamma_{0}$ of $\partial G$ ) evolving in $G$ vanishes almost surely, provided that its normal derivative equals 0 in $\Gamma_{0} \times(0, T), P$-a.s. Compared with the result in Theorem 1.2, the results in reference $[15,28]$ do not need the homogeneous Dirichlet boundary condition. However, they have to utilize the information of the solution in the whole time duration $(0, T)$. On the other hand, Theorem 1.2 means that the solution vanishes almost surely if it vanishes in $G_{0} \times\left\{T_{0}\right\}$. That is, we need only the information of the solution to (1.1) in $G_{0}$ at any fixed positive time constant $T_{0}$ rather than the whole time duration.

As an application of Theorem 1.2, we give an approximate controllability result for backward stochastic heat equations. We denote by $\left\{\mathcal{W}_{t}\right\}_{t \geq 0}$ the natural filtration generated by $\{B(t)\}_{t \geq 0}$, which is augmented by all the $P$-null sets. Let $H$ be a Banach space. We denote by $L_{\mathcal{W}}^{2}(0, T ; H)$ the Banach space of all $H$-valued and $\left\{\mathcal{W}_{t}\right\}_{t \geq 0}$-adapted processes $X(\cdot)$ with $\mathbb{E}\left(\|X(\cdot)\|_{L^{2}(0, T ; H)}^{2}\right)<\infty$; by $L_{\mathcal{W}}^{\infty}(0, T ; H)$ the Banach space consisting of all $H$-valued $\left\{\mathcal{W}_{t}\right\}_{t \geq 0}$-adapted bounded processes and by $L_{\mathcal{W}}^{2}(\Omega ; C([0, T] ; H))$ the Banach space consisting of all $H$-valued $\left\{\mathcal{W}_{t}\right\}_{t \geq 0}$-adapted continuous processes $X(\cdot)$ such that $\mathbb{E}\left(\|X(\cdot)\|_{C([0, T] ; H)}^{2}\right)<\infty$. All the above spaces are endowed with the canonical norms.

Assume that $\partial G$ is $C^{2}$. Consider the following controlled backward stochastic heat equation

$$
\begin{cases}\mathrm{d} z+\Delta z \mathrm{~d} t=a_{1} z \mathrm{~d} t+b_{1} Z \mathrm{~d} t+h \mathrm{~d} t+\chi_{E_{1}} \chi_{G_{0}} f \mathrm{~d} t+Z \mathrm{~d} B(t) & \text { in } G \times(0, T), \\ z=0 & \text { on } \partial G \times(0, T), \\ z(T)=z_{T} & \text { in } G .\end{cases}
$$

Here $E_{1} \subset[0, T]$ is a Lebesgue measurable subset with positive measure, $z_{T} \in L^{2}\left(\Omega, \mathcal{W}_{T}, P ; L^{2}(G)\right), a_{1} \in$ $L_{\mathcal{W}}^{\infty}\left(0, T ; L^{\infty}(G)\right), b_{1} \in L_{\mathcal{W}}^{\infty}\left(0, T ; W^{1, \infty}(G)\right), h \in L_{\mathcal{W}}^{2}\left(0, T ; L^{2}(G)\right)$, and $f \in L_{\mathcal{W}}^{2}\left(0, T ; L^{2}(G)\right)$ is the control.

Following the duality analysis in [29], one can show that (1.9) admits a unique solution

$$
(z, Z) \in\left[L_{\mathcal{W}}^{2}\left(\Omega ; C\left([0, T] ; L^{2}(G)\right)\right) \cap L_{\mathcal{W}}^{2}\left(0, T ; H_{0}^{1}(G)\right)\right] \times L_{\mathcal{W}}^{2}\left(0, T ; L^{2}(G)\right) .
$$

Definition 1.8. System (1.9) is approximately controllable if for any $z_{T} \in L^{2}\left(\Omega, \mathcal{W}_{T}, P ; L^{2}(G)\right)$, any $z_{0} \in$ $L^{2}(G)$ and any $\varepsilon>0$, there exists a control $f \in L_{\mathcal{W}}^{2}\left(0, T ; L^{2}(G)\right)$ such that the solution to the system (1.9) with terminal state $z_{T}$ and control $f$ satisfying that $\left\|z(0)-z_{0}\right\|_{L^{2}(G)} \leq \varepsilon$. 
Making use of Theorem 1.2, we obtain the following result.

Theorem 1.9. System (1.9) is approximately controllable.

The approximate controllability for deterministic heat equations is a classical topic in control theory and almost well understood now. We refer the readers to $[2,5,7,8]$ and the rich references therein for this topic. However, the approximate controllability problems for forward and backward stochastic heat equations are quite open. Some special cases in which the approximate controllability problem for stochastic heat equations can be reduced to the same problem for deterministic ones were studied in reference $[1,9,19,24]$. In reference [17], the author shows that the null controllability of a stochastic heat equation does not imply its approximate controllability. As a direct consequence of the observability estimate in reference [25], one can deduce the approximate controllability of backward stochastic heat equations evolving in bounded domains. Compared with the result in reference [25], in the present paper, the control acts only on a measurable subset of $[0, T]$ rather than the whole interval and the domain $G$ can be unbounded.

As another application of Theorem 1.6, we have the following observability inequality.

Theorem 1.10. Assume that Condition 1.4 holds. Let $E \subset(0, T)$ be a subset with positive Lebesgue measure and $G_{0}$ be a nonempty open subset of $G$. Then any solution y to (1.1) satisfies the estimate

$$
\|y(T)\|_{L_{\mathcal{F}_{T}}^{2}\left(\Omega ; L^{2}(G)\right)}^{2} \leq C \mathbb{E} \int_{E} \int_{G_{0}} y^{2}(x, t) \mathrm{d} x \mathrm{~d} t .
$$

Observability inequalities play important roles in the study of controllability problems and state observation problems (see $[27,30]$ for example). The observability estimate for heat equations with lower order potentials depending both on $x$ and $t$ was first proved in reference [10] by a global Carleman estimate. In that work, the integral with respect to $t$ is over $[0, T]$ rather than only a measurable set. The inequality in the form of (1.10) was obtained in reference [21] and was used to get the null controllability and the Bang-bang principle for the time optimal control of heat equations.

As an immediate consequence of Theorem 1.10, we can get the null controllability of backward stochastic heat equations. Let us first recall the following definition.

Definition 1.11. System (1.9) is said to be null controllable if for any $z_{T} \in L^{2}\left(\Omega, \mathcal{W}_{T}, P ; L^{2}(G)\right)$, there exists a control $f \in L_{\mathcal{W}}^{2}\left(0, T ; L^{2}(G)\right)$ such that the solution of the system (1.9) with terminal state $z_{T}$ and control $f$ satisfying that $z(0)=0$.

We have the following result.

Theorem 1.12. Assume that $h=0$ in (1.9). Then (1.9) is null controllable, provided that $G$ is bounded and convex.

The null controllability for heat equations is also a classical topic in the control theory of partial differential equations. There are a large of literatures about it (see $[6,8,10,14]$ and the references therein). Similar to the case of approximate controllability problems, the study of null controllability problems for forward and backward stochastic heat equations are very uncomplete. In reference [25], the authors got the null controllability of backward stochastic heat equations evolving in bounded domains. Compared with the result in reference [25], in the present paper, the control acts only on a measurable subset of $[0, T]$ rather than the whole interval but the domain $G$ should be convex.

The rest of this paper is organized as follows: in Section 2, we introduce some standing notations and derive some lemmas as preliminaries for the proof of Theorem 1.2. Section 3 is devoted to the proof of Theorem 1.2. Section 4 is devoted to the proof of Theorem 1.6. In Section 5, we prove the observability result, i.e., Theorem 1.10. Finally, Section 6 is devoted to the proof of Theorems 1.9 and 1.12. 


\section{SOME PRELIMINARIES}

This section is devoted to some preliminaries for the proof of Theorem 1.2. In what follows, for simplicity of notations, we adopt $\vartheta\left(\cdot, x, x_{0}\right)=\exp \left(-\left|x-x_{0}\right|^{2} / 4(\cdot)\right)$ and $\|a\|$. , $\|b\|$. for $\|a\|_{L_{\mathcal{F}}^{\infty}\left(0, T ; L^{\infty}(\cdot)\right)},\|b\|_{L_{\mathcal{F}}^{\infty}\left(0, T ; W^{1, \infty}(\cdot)\right)}$ respectively. We fix a point $x_{0} \in G_{0}$. For $\lambda>0$, define

$$
K(x, t) \triangleq(T-t+\lambda)^{-\frac{n}{2}} \vartheta\left(T-t+\lambda, x, x_{0}\right), \quad(x, t) \in G \times[0, T] .
$$

It is clear that

$$
\left\{\begin{array}{l}
K_{t}+\Delta K=0, \quad \nabla K=-\frac{x-x_{0}}{2(T-t+\lambda)} K, \\
\Delta K=\frac{-n}{2(T-t+\lambda)} K+\frac{\left|x-x_{0}\right|^{2}}{4(T-t+\lambda)^{2}} K, \\
K_{x_{i} x_{j}}=\frac{\left(x_{i}-x_{0 i}\right)\left(x_{j}-x_{0 j}\right)}{4(T-t+\lambda)^{2}} K, \quad i \neq j .
\end{array}\right.
$$

Let $\varphi$ be a $C^{\infty}$ function with support $\widetilde{G} \subset G$ and $\Phi=\varphi y$. Let $F=a \Phi-y \Delta \varphi-2 \nabla \varphi \cdot \nabla y$. It follows that

$$
\mathrm{d} \Phi-\Delta \Phi \mathrm{d} t=F \mathrm{~d} t+b \Phi \mathrm{d} B .
$$

For $t \in[0, T]$, we put

$$
\left\{\begin{array}{l}
H(t)=\mathbb{E} \int_{G}|\Phi(x, t)|^{2} K(x, t) \mathrm{d} x, \\
D(t)=\mathbb{E} \int_{G}|\nabla \Phi(x, t)|^{2} K(x, t) \mathrm{d} x, \\
N(t)=\frac{2 D(t)}{H(t)} \text { provided that } H(t) \neq 0 .
\end{array}\right.
$$

Throughout this section, we always work under the assumption $H(\cdot) \neq 0$.

Lemma 2.1. For the function $H(\cdot)$ defined in (2.4), involving the solution y to equation (1.1), it holds that

$$
H^{\prime}(t)=-2 D(t)+2 \mathbb{E} \int_{G} \Phi F K \mathrm{~d} x+\mathbb{E} \int_{G} b^{2} \Phi^{2} K \mathrm{~d} x .
$$

Proof. Following Itô's formula and noticing that $\Phi$ has zero boundary condition, we have that

$$
\begin{aligned}
H(t)-H(s)= & 2 \mathbb{E} \int_{s}^{t} \int_{s} \Phi \mathrm{d} \Phi K \mathrm{~d} x+\mathbb{E} \int_{s}^{t} \int_{G}(\mathrm{~d} \Phi)^{2} K \mathrm{~d} x+\mathbb{E} \int_{s}^{t} \int_{G} \Phi^{2} K_{t} \mathrm{~d} x \mathrm{~d} \tau \\
= & 2 \mathbb{E} \int_{s}^{t} \int_{G} \Phi[\Delta \Phi \mathrm{d} \tau+F \mathrm{~d} \tau+b \Phi \mathrm{d} B(\tau)] K \mathrm{~d} x+\mathbb{E} \int_{s}^{t} \int_{G}(\mathrm{~d} \Phi)^{2} K \mathrm{~d} x-\mathbb{E} \int_{s}^{t} \int_{G} \Phi^{2} \Delta K \mathrm{~d} x \mathrm{~d} \tau \\
= & 2 \mathbb{E} \int_{s}^{t} \int_{G} \Phi \Delta \Phi K \mathrm{~d} x \mathrm{~d} \tau-\mathbb{E} \int_{s}^{t} \int_{G} \Phi^{2} \Delta K \mathrm{~d} x \mathrm{~d} t+2 \mathbb{E} \int_{s}^{t} \int_{G} \Phi F K \mathrm{~d} x \mathrm{~d} \tau \\
& +\mathbb{E} \int_{s}^{t} \int_{G}(\mathrm{~d} \Phi)^{2} K \mathrm{~d} x+2 \mathbb{E} \int_{s}^{t} \int_{G} b \Phi^{2} K \mathrm{~d} x \mathrm{~d} B(\tau) \\
= & -2 \mathbb{E} \int_{s}^{t} \int_{G}|\nabla \Phi|^{2} K \mathrm{~d} x \mathrm{~d} \tau+2 \mathbb{E} \int_{s}^{t} \int_{G} \Phi F K \mathrm{~d} x \mathrm{~d} \tau+\mathbb{E} \int_{s}^{t} \int_{G}(\mathrm{~d} \Phi)^{2} K \mathrm{~d} x \\
= & -2 \mathbb{E} \int_{s}^{t} \int_{G}|\nabla \Phi|^{2} K \mathrm{~d} x \mathrm{~d} \tau+2 \mathbb{E} \int_{s}^{t} \int_{G} \Phi F K \mathrm{~d} x \mathrm{~d} \tau+\mathbb{E} \int_{s}^{t} \int_{G} b^{2} \Phi^{2} K \mathrm{~d} x \mathrm{~d} \tau
\end{aligned}
$$

As a result, it is easy to derive from (2.6) that

$$
H^{\prime}(t)=-2 D(t)+2 \mathbb{E} \int_{G} \Phi F K \mathrm{~d} x+\mathbb{E} \int_{G} b^{2} \Phi^{2} K \mathrm{~d} x .
$$

Thus, we complete the proof. 
Lemma 2.2. For $0 \leq s<t \leq T$, it follows that

$$
N(t)-N(s) \leq \int_{s}^{t}\left(\frac{1}{T-\tau+\lambda}+2\|b\|_{\widetilde{G}}^{2}\right) N(\tau) \mathrm{d} \tau+2\|b\|_{\widetilde{G}}^{2}(t-s)+\int_{s}^{t} \frac{\mathbb{E} \int_{G} F^{2} K \mathrm{~d} x}{H} \mathrm{~d} \tau .
$$

Proof. First, we have that

$$
\begin{aligned}
D(t)-D(s)= & 2 \mathbb{E} \int_{s}^{t} \int_{G} \Phi \mathrm{d} \Phi K \mathrm{~d} x+\mathbb{E} \int_{s}^{t} \int_{G}(\mathrm{~d} \Phi)^{2} K \mathrm{~d} x+\mathbb{E} \int_{s}^{t} \int_{G} \Phi^{2} K_{t} \mathrm{~d} x \mathrm{~d} \tau \\
= & 2 \mathbb{E} \int_{s}^{t} \int_{G} \Phi \mathrm{d} \Phi K \mathrm{~d} x+\mathbb{E} \int_{s}^{t} \int_{G}(\mathrm{~d} \Phi)^{2} K \mathrm{~d} x-\mathbb{E} \int_{s}^{t} \int_{G} \Phi^{2} \Delta K \mathrm{~d} x \mathrm{~d} \tau \\
= & 2 \mathbb{E} \int_{s}^{t} \int_{G} \Phi \mathrm{d} \Phi K \mathrm{~d} x+\mathbb{E} \int_{s}^{t} \int_{G}(\mathrm{~d} \Phi)^{2} K \mathrm{~d} x \\
& -\mathbb{E} \int_{s}^{t} \int_{G} \nabla \cdot\left(\Phi^{2} \nabla K\right) \mathrm{d} x \mathrm{~d} \tau+\mathbb{E} \int_{s}^{t} \int_{G} \nabla\left(\Phi^{2}\right) \cdot \nabla K \mathrm{~d} x \mathrm{~d} \tau \\
= & 2 \mathbb{E} \int_{s}^{t} \int_{G} \Phi(\Delta \Phi+F) K \mathrm{~d} x \mathrm{~d} \tau+2 \mathbb{E} \int_{s}^{t} \int_{G} b \Phi^{2} K \mathrm{~d} x \mathrm{~d} B(\tau)+\mathbb{E} \int_{s}^{t} \int_{G} b^{2} \Phi^{2} K \mathrm{~d} x \mathrm{~d} \tau \\
& -2 \mathbb{E} \int_{s}^{t} \int_{G} \Phi \frac{x-x_{0}}{2(T-\tau+\lambda)} \cdot \nabla \Phi K \mathrm{~d} x \mathrm{~d} \tau \\
= & 2 \mathbb{E} \int_{s}^{t} \int_{G} \Phi\left(\Delta \Phi+\frac{F}{2}-\frac{x-x_{0}}{2(T-\tau+\lambda)} \cdot \nabla \Phi\right) K \mathrm{~d} x \mathrm{~d} \tau \\
& +\mathbb{E} \int_{s}^{t} \int_{G} \Phi F K \mathrm{~d} x \mathrm{~d} \tau+\mathbb{E} \int_{s}^{t} \int_{G} b^{2} \Phi^{2} K \mathrm{~d} x \mathrm{~d} \tau .
\end{aligned}
$$

Next, following some straightforward calculations, we have that

$$
\begin{aligned}
D(t)-D(s)= & 2 \mathbb{E} \int_{s}^{t} \int_{s} \nabla \Phi \mathrm{d} \nabla \Phi K \mathrm{~d} x+\mathbb{E} \int_{s}^{t} \int_{G}|\mathrm{~d} \nabla \Phi|^{2} K \mathrm{~d} x-\mathbb{E} \int_{s}^{t} \int_{G}|\nabla \Phi|^{2} \Delta K \mathrm{~d} x \mathrm{~d} \tau \\
= & -2 \mathbb{E} \int_{s}^{t} \int_{G} \mathrm{~d} \Phi \Delta \Phi K \mathrm{~d} x+2 \mathbb{E} \int_{s}^{t} \int_{G} \mathrm{~d} \Phi \frac{x-x_{0}}{2(T-\tau+\lambda)} \cdot \nabla \Phi K \mathrm{~d} x \\
& +\mathbb{E} \int_{s}^{t} \int_{G}|\mathrm{~d} \nabla \Phi|^{2} K \mathrm{~d} x-2 \mathbb{E} \int_{s}^{t} \int_{G} \Delta \Phi \nabla \Phi \cdot \nabla K \mathrm{~d} x \mathrm{~d} \tau-2 \sum_{i=1}^{n} \mathbb{E} \int_{s}^{t} \int_{G} \Phi x_{i} \nabla \Phi \cdot \nabla K_{x_{i}} \mathrm{~d} x \mathrm{~d} \tau \\
= & -2 \mathbb{E} \int_{s}^{t} \int_{G} \mathrm{~d} \Phi \Delta \Phi K \mathrm{~d} x+2 \mathbb{E} \int_{s}^{t} \int_{G}(\mathrm{~d} \Phi+\Delta \Phi \mathrm{d} \tau) \frac{x-x_{0}}{2(T-\tau+\lambda)} \cdot \nabla \Phi K \mathrm{~d} x \\
& +\mathbb{E} \int_{s}^{t} \int_{G}|\mathrm{~d} \nabla \Phi|^{2} K \mathrm{~d} x+\mathbb{E} \int_{s}^{t} \frac{1}{T-\tau+\lambda} \int_{G}|\nabla \Phi|^{2} K \mathrm{~d} x \mathrm{~d} \tau \\
& -2 \mathbb{E} \int_{s}^{t} \int_{G}\left(\frac{x-x_{0}}{2(T-\tau+\lambda)} \cdot \nabla \Phi\right)^{2} K \mathrm{~d} x \mathrm{~d} \tau .
\end{aligned}
$$

In the above equality, no boundary terms appear due to the fact that $\Phi$ vanishes in a neighborhood of the boundary of $G$. 
Aided by equation (2.3) and regrouping the right hand side of the above equality follows that

$$
\begin{aligned}
D(t)-D(s)= & \mathbb{E} \int_{s}^{t} \frac{1}{T-\tau+\lambda} \int_{G}|\nabla \Phi|^{2} K \mathrm{~d} x \mathrm{~d} \tau+\mathbb{E} \int_{s}^{t} \int_{G}|\mathrm{~d} \nabla \Phi|^{2} K \mathrm{~d} x \\
& -2\left[\mathbb{E} \int_{s}^{t} \int_{G}\left(\frac{x-x_{0}}{2(T-\tau+\lambda)} \cdot \nabla \Phi\right)^{2} K \mathrm{~d} x \mathrm{~d} \tau+\mathbb{E} \int_{s}^{t} \int_{G}(\Delta \Phi+F) \Delta \Phi K \mathrm{~d} x \mathrm{~d} \tau\right. \\
& \left.-\mathbb{E} \int_{s}^{t} \int_{G}(2 \Delta \Phi+F) \frac{x-x_{0}}{2(T-\tau+\lambda)} \cdot \nabla \Phi K \mathrm{~d} x \mathrm{~d} \tau\right] \\
& -2 \mathbb{E} \int_{s}^{t} \int_{G} b \Phi \Delta \Phi K \mathrm{~d} x \mathrm{~d} B(\tau)+2 \mathbb{E} \int_{s}^{t} \int_{G} b \Phi \frac{x-x_{0}}{2(T-\tau+\lambda)} \cdot \nabla \Phi K \mathrm{~d} x \mathrm{~d} B(\tau) \\
= & -2 \mathbb{E} \int_{s}^{t} \int_{G}\left[\Delta \Phi+\frac{F}{2}-\frac{x-x_{0}}{2(T-\tau+\lambda)} \cdot \nabla \Phi\right]^{2} K \mathrm{~d} x \mathrm{~d} \tau+\frac{1}{2} \mathbb{E} \int_{s}^{t} \int_{G} F^{2} K \mathrm{~d} x \mathrm{~d} \tau \\
& +\mathbb{E} \int_{s}^{t} \frac{1}{T-\tau+\lambda} \int_{G}|\nabla \Phi|^{2} K \mathrm{~d} x \mathrm{~d} \tau+\mathbb{E} \int_{s}^{t} \int_{G}|\nabla(b \Phi)|^{2} K \mathrm{~d} x \mathrm{~d} \tau \\
& -2 \mathbb{E} \int_{s}^{t} \int_{G} b \Phi \Delta \Phi K \mathrm{~d} x \mathrm{~d} B(\tau)+2 \mathbb{E} \int_{s}^{t} \int_{G} b \Phi \frac{x-x_{0}}{2(T-\tau+\lambda)} \cdot \nabla \Phi K \mathrm{~d} x \mathrm{~d} B(\tau) .
\end{aligned}
$$

On the other hand, we have that

$$
\begin{aligned}
D(t) & =\mathbb{E} \int_{G}|\nabla \Phi|^{2} K \mathrm{~d} x \\
& =\mathbb{E} \int_{G} \nabla \cdot(\Phi \nabla \Phi K) \mathrm{d} x-\mathbb{E} \int_{G} \Phi \Delta \Phi K \mathrm{~d} x-\mathbb{E} \int_{G} \Phi \nabla \Phi \cdot \nabla K \mathrm{~d} x \\
& =-\mathbb{E} \int_{G} \Phi \Delta \Phi \mathrm{d} x+\mathbb{E} \int_{G} \Phi \nabla \Phi \cdot \frac{x-x_{0}}{2(T-t+\lambda)} K \mathrm{~d} x \\
& =-\mathbb{E} \int_{G} \Phi\left(\Delta \Phi+\frac{F}{2}-\frac{x-x_{0}}{2(T-t+\lambda)} \cdot \nabla \Phi\right) K \mathrm{~d} x+\frac{1}{2} \mathbb{E} \int_{G} F \Phi K \mathrm{~d} x .
\end{aligned}
$$

Based on (2.5), (2.9) and (2.10), for any $0 \leq s<t \leq T$, it follows that

$$
\begin{aligned}
N(t)-N(s)= & 2 \int_{s}^{t} \frac{H \mathrm{~d} D-D \mathrm{~d} H}{H^{2}} \\
= & -\int_{s}^{t} \frac{4}{H} \mathbb{E} \int_{G}\left[\Delta \Phi+\frac{F}{2}-\frac{x-x_{0}}{2(T-\tau+\lambda)} \cdot \nabla \Phi\right]^{2} K \mathrm{~d} x \mathrm{~d} \tau \\
& +\int_{s}^{t} \frac{4}{H^{2}}\left[\mathbb{E} \int_{G} \Phi\left(\Delta \Phi+\frac{F}{2}-\frac{x-x_{0}}{2(T-\tau+\lambda)} \cdot \nabla \Phi\right) K \mathrm{~d} x\right]^{2} \mathrm{~d} \tau \\
& -\int_{s}^{t} \frac{1}{H^{2}}\left(\mathbb{E} \int_{G} F K \mathrm{~d} x\right)^{2} \mathrm{~d} \tau-\int_{s}^{t} \frac{2 D}{H} \mathbb{E} \int_{G} b^{2} \Phi^{2} K \mathrm{~d} x \mathrm{~d} \tau \\
& +\int_{s}^{t} \frac{2}{H} \mathbb{E} \int_{G}|\nabla(b \Phi)|^{2} K \mathrm{~d} x \mathrm{~d} \tau+\int_{s}^{t} \frac{1}{T-\tau+\lambda} \frac{2 D}{H} \mathrm{~d} \tau+\int_{s}^{t} \frac{\mathbb{E} \int_{G} F^{2} K \mathrm{~d} x}{H} \mathrm{~d} \tau
\end{aligned}
$$

Applying Cauchy-Schwarz's inequality to the second term of the right hand side of the above equality and noticing that $\Phi=\varphi y$ is supported in $\widetilde{G}$, we arrive at that

$$
\begin{aligned}
N(t)-N(s) & \leq \int_{s}^{t} \frac{N(\tau)}{T-\tau+\lambda} \mathrm{d} \tau+\int_{s}^{t} \frac{2}{H} \mathbb{E} \int_{G}|\nabla(b \Phi)|^{2} K \mathrm{~d} x \mathrm{~d} \tau+\int_{s}^{t} \frac{\mathbb{E} \int_{G} F^{2} K \mathrm{~d} x}{H} \mathrm{~d} \tau \\
& \leq \int_{s}^{t}\left(\frac{1}{T-\tau+\lambda}+2\|b\|_{\widetilde{G}}^{2}\right) N(\tau) \mathrm{d} \tau+2\|b\|_{\widetilde{G}}^{2}(t-s)+\int_{s}^{t} \frac{\mathbb{E} \int_{G} F^{2} K \mathrm{~d} x}{H} \mathrm{~d} \tau .
\end{aligned}
$$


As a result, we complete the proof.

At last, we introduce the following backward uniqueness for solutions to (1.1).

Lemma 2.3. Assume that Condition 1.4 holds. Let $y$ be a solution to equation (1.1). If $y(T)=0$ in $G$, P-a.s., then $y(t)=0$ in $G, P$-a.s., for all $t \in[0, T]$.

Remark 2.4. Lemma 2.3 was first proved in [18] (see [18], Cor. 1.1 for the details) for bounded domain $G$. By a very similar but lengthy argument, the same result can be obtained for unbounded $G$. Hence, we omit the detailed proof here.

\section{Proof of Theorem 1.2}

This section is devoted to the Proof of Theorem 1.2.

Let $B_{r_{i}}, i=1,2,3,4$ be balls with center $x_{0}$ and radius $r_{i}$ respectively. These balls have the property $B_{r}=$ $B_{r_{1}} \subset G_{0}, B_{r_{j}} \subset \subset B_{r_{j+1}} \subset \subset G(j=1,2,3)$. We also choose a special truncated function $\varphi$ for the transformation $\Phi=\varphi y$ such that $\varphi$ satisfies the properties

$$
\operatorname{supp} \varphi \subset B_{r_{4}} \text { and } \varphi=1 \text { in } B_{r_{3}} .
$$

We borrow some ideas from [20,22]. In those papers, the author defined functions like $N, H, D$ in our paper and discussed the relations among them to obtain a quantitative unique continuation for deterministic heat equations involving in a bounded domain with Dirichlet boundary condition. But for the stochastic settings, though the idea can be borrowed, the case is much different for that there are random terms involved.

Proof of Theorem 1.2. Without loss of generality and for the simplicity of the notations, we only consider the case $T_{0}=T$ here. The general case can be handled similarly.

We first prove that if $y(x, T)=0, P$-a.s. in $B_{r_{1}}$, then it also vanishes in $B_{r_{2}}$. We do this by contradiction argument. In fact, if this claim does not hold, then $\mathbb{E} \int_{B_{r_{2}}} y^{2}(x, T) \mathrm{d} x \neq 0$. Therefore, from the continuity of the solution $y$ of (1.1) with respect to $t$, there must be some small positive constant $\varepsilon$, such that

$$
\mathbb{E} \int_{B_{r_{2}}} y^{2}(x, t) \mathrm{d} x \neq 0 \quad \text { for all } t \in[T-2 \varepsilon, T] .
$$

Let us consider the following inequality:

$$
\int_{G}\left|\nabla\left[\Phi(x, T) \vartheta\left(2 \lambda, x, x_{0}\right)\right]\right|^{2} \mathrm{~d} x \geq 0,
$$

from which we can obtain some information about $N(T)$. By some straightforward computations, one has that

$$
\begin{aligned}
\int_{G}\left|\nabla \Phi(x, T)-\frac{x-x_{0}}{4 \lambda} \Phi(x, T)\right|^{2} \vartheta\left(\lambda, x, x_{0}\right) \mathrm{d} x \leq & \int_{G}|\nabla \Phi(x, T)|^{2} \vartheta\left(\lambda, x, x_{0}\right) \mathrm{d} x+\int_{G} \frac{\left|x-x_{0}\right|^{2}}{16 \lambda^{2}} \Phi^{2}(x, T) \vartheta\left(\lambda, x, x_{0}\right) \mathrm{d} x \\
& -2 \int_{G} \frac{x-x_{0}}{4 \lambda} \cdot \nabla \Phi(x, T) \Phi(x, T) \vartheta\left(\lambda, x, x_{0}\right) \mathrm{d} x \\
\leq & \int_{G}|\nabla \Phi(x, T)|^{2} \vartheta\left(\lambda, x, x_{0}\right) \mathrm{d} x+\int_{G} \frac{\left|x-x_{0}\right|^{2}}{16 \lambda^{2}} \Phi^{2}(x, T) \vartheta\left(\lambda, x, x_{0}\right) \mathrm{d} x \\
& +\frac{n}{4 \lambda} \int_{G} \Phi^{2}(x, T) \vartheta\left(\lambda, x, x_{0}\right) \mathrm{d} x \\
& -\int_{G} \frac{\left|x-x_{0}\right|^{2}}{8 \lambda^{2}} \Phi^{2}(x, T) \vartheta\left(\lambda, x, x_{0}\right) \mathrm{d} x .
\end{aligned}
$$

In the above process, we adopt integration by parts in the last step. It is easy to find that

$$
\int_{G} \frac{\left|x-x_{0}\right|^{2}}{8 \lambda} \Phi^{2}(x, T) \vartheta\left(\lambda, x, x_{0}\right) \mathrm{d} x \leq \frac{n}{2} \int_{G} \Phi^{2}(x, T) \vartheta\left(\lambda, x, x_{0}\right) \mathrm{d} x+2 \lambda \int_{G}|\nabla \Phi(x, T)|^{2} \vartheta\left(\lambda, x, x_{0}\right) \mathrm{d} x .
$$


Taking mathematical expectation from both sides of (3.2) and noticing that

$$
\begin{aligned}
\mathbb{E} \int_{G}|\nabla \Phi(x, T)|^{2} \vartheta\left(\lambda, x, x_{0}\right) \mathrm{d} x & =\frac{\mathbb{E} \int_{G}|\nabla \Phi(x, T)|^{2} \vartheta\left(\lambda, x, x_{0}\right) \mathrm{d} x}{\mathbb{E} \int_{G} \Phi^{2}(x, T) \vartheta\left(\lambda, x, x_{0}\right) \mathrm{d} x}\left[\mathbb{E} \int_{G} \Phi^{2}(x, T) \vartheta\left(\lambda, x, x_{0}\right) \mathrm{d} x\right] \\
& =\frac{1}{2} N(T) \mathbb{E} \int_{G} \Phi^{2}(x, T) \vartheta\left(\lambda, x, x_{0}\right) \mathrm{d} x,
\end{aligned}
$$

we have that

$$
\begin{aligned}
\mathbb{E} \int_{G}\left|x-x_{0}\right|^{2} \Phi^{2}(x, T) \vartheta\left(\lambda, x, x_{0}\right) \mathrm{d} x & \leq 8 \lambda\left(\lambda N(T)+\frac{n}{2}\right) \mathbb{E} \int_{G} \Phi^{2}(x, T) \vartheta\left(\lambda, x, x_{0}\right) \mathrm{d} x \\
& \leq 8 \lambda\left(\lambda N(T)+\frac{n}{2}\right)\left[\mathbb{E} \int_{B_{r}} \Phi^{2}(x, T) \vartheta\left(\lambda, x, x_{0}\right) \mathrm{d} x\right. \\
& \left.+\mathbb{E} \int_{G \backslash B_{r}} \Phi^{2}\left(x, T_{0}\right) \vartheta\left(\lambda, x, x_{0}\right) \mathrm{d} x\right] \\
& \leq 8 \lambda\left(\lambda N(T)+\frac{n}{2}\right)\left[\mathbb{E} \int_{B_{r}} \Phi^{2}\left(x, T_{0}\right) \vartheta\left(\lambda, x, x_{0}\right) \mathrm{d} x\right. \\
& \left.+\frac{1}{r^{2}} \mathbb{E} \int_{G \backslash B_{r}}\left|x-x_{0}\right|^{2} \Phi^{2}(x, T) \vartheta\left(\lambda, x, x_{0}\right) \mathrm{d} x\right]
\end{aligned}
$$

From this inequality, we immediately obtain that

$\left[1-\frac{8 \lambda}{r^{2}}\left(\lambda N(T)+\frac{n}{2}\right)\right] \mathbb{E} \int_{G}\left|x-x_{0}\right|^{2} \Phi^{2}(x, T) \vartheta\left(\lambda, x, x_{0}\right) \mathrm{d} x \leq 8 \lambda\left(\lambda N(T)+\frac{n}{2}\right) \mathbb{E} \int_{B_{r}} \Phi^{2}(x, T) \vartheta\left(\lambda, x, x_{0}\right) \mathrm{d} x$.

Now, (3.4) shows that we should give an estimate for $\lambda N(T)$.

Based on Lemma 2.2 and Gronwall's inequality, it follows for $t \in[T-2 \varepsilon, T]$ that

$$
N(T) \leq\left[N(t)+2 T\|b\|_{B_{r_{4}}}^{2}+\int_{t}^{T} \frac{\mathbb{E} \int_{G} F^{2} K \mathrm{~d} x}{H} \mathrm{~d} \tau\right] \exp \left[\int_{t}^{T}\left(\frac{1}{T-\tau+\lambda}+2\|b\|_{B_{r_{4}}}^{2}\right) \mathrm{d} \tau\right],
$$

which implies that

$$
\begin{aligned}
\lambda N(T) \leq & (T+\lambda) \exp \left(2 T\|b\|_{B_{r_{4}}}^{2}\right) N(t) \\
& +(T+\lambda) \exp \left(2 T\|b\|_{B_{r_{4}}}^{2}\right)\left(2 T\|b\|_{B_{r_{4}}}^{2}+\int_{t}^{T} \frac{\mathbb{E} \int_{G} F^{2} K \mathrm{~d} x}{H} \mathrm{~d} \tau\right) .
\end{aligned}
$$

Integrating (3.5) with respect to $t$ over $[T-2 \varepsilon, T-\varepsilon]$, we find that

$$
\begin{aligned}
\varepsilon \lambda N(T) \leq & (T+\lambda) \exp \left(2 T\|b\|_{B_{r_{4}}}^{2}\right) \int_{T-2 \varepsilon}^{T-\varepsilon} N(t) \mathrm{d} t \\
& +\varepsilon(T+\lambda) \exp \left(2 T\|b\|_{B_{r_{4}}}^{2}\right)\left(2 T\|b\|_{B_{r_{4}}}^{2}+\int_{T-2 \varepsilon}^{T} \frac{\mathbb{E} \int_{G} F^{2} K \mathrm{~d} x}{H} \mathrm{~d} \tau\right) .
\end{aligned}
$$

By Lemma 2.1, we have that

$$
\int_{T-2 \varepsilon}^{T-\varepsilon} N(t) \mathrm{d} t \leq \ln \frac{H(T-2 \varepsilon)}{H(T-\varepsilon)}+2 \int_{T-2 \varepsilon}^{T-\varepsilon} \frac{\mathbb{E} \int_{G} \Phi F K \mathrm{~d} x}{H} \mathrm{~d} t+\varepsilon\|b\|_{B_{r_{4}}}^{2} .
$$


From (3.6) and (3.7), we see that

$$
\begin{aligned}
\lambda N(T) \leq & \frac{T+\lambda}{\varepsilon} \exp \left(2 T\|b\|_{B_{r_{4}}}^{2}\right)\left[\ln \frac{H(T-2 \varepsilon)}{H(T-\varepsilon)}+2 \int_{T-2 \varepsilon}^{T-\varepsilon} \frac{\mathbb{E} \int_{G} \Phi F K \mathrm{~d} x}{H} \mathrm{~d} t \varepsilon(1+2 T)\|b\|_{B_{r_{4}}}^{2}\right. \\
& \left.+\varepsilon \int_{0}^{T} \frac{\mathbb{E} \int_{G} F^{2} K \mathrm{~d} x}{H} \mathrm{~d} \tau\right] \\
\leq & \frac{T+\lambda}{\varepsilon} \exp \left(2 T\|b\|_{B_{r_{4}}}^{2}\right)\left[\ln \frac{H(T-2 \varepsilon)}{H(T-\varepsilon)}+\varepsilon+\varepsilon(1+2 T)\|b\|_{B_{r_{4}}}^{2}\right. \\
& \left.+(\varepsilon+1) \int_{T-2 \varepsilon}^{T} \frac{\mathbb{E} \int_{G} F^{2} K \mathrm{~d} x}{H} \mathrm{~d} t\right] .
\end{aligned}
$$

For brevity of notations, we denote by $\mathcal{A}(\lambda)$ the right hand side of (3.8). We claim that the term involving $F$ in $\mathcal{A}(\lambda)$ are uniformly bounded with respect to $\lambda \in[0,1]$ for fixed $y$ solving (1.1).

Recalling that $\Phi=\varphi y$ and $F=a \varphi y-2 \nabla \varphi \cdot \nabla y-y \Delta \varphi$, we get that

$$
\begin{aligned}
\int_{T-2 \varepsilon}^{T} \frac{\mathbb{E} \int_{G} F^{2} K \mathrm{~d} x}{H} \mathrm{~d} t & \leq 2 T\|a\|_{B_{r_{4}}}^{2}+8 \int_{T-2 \varepsilon}^{T} \frac{\mathbb{E} \int_{G}|\nabla \varphi|^{2}|\nabla y|^{2} K \mathrm{~d} x}{H} \mathrm{~d} t+2 \int_{T-2 \varepsilon}^{T} \frac{\mathbb{E} \int_{G}|\Delta \varphi|^{2} y^{2} K}{H} \mathrm{~d} t \\
\leq & 2 T\|a\|_{B_{r_{4}}}^{2}+8 \int_{T-2 \varepsilon}^{T} \frac{\mathbb{E} \int_{G \backslash B_{r_{3}}}|\nabla \varphi|^{2}|\nabla y|^{2} K \mathrm{~d} x}{\mathbb{E} \int_{B_{r_{2}}} y^{2} K \mathrm{~d} x} \mathrm{~d} t+2 \int_{T-2 \varepsilon}^{T} \frac{\mathbb{E} \int_{G \backslash B_{r_{3}}}|\Delta \varphi|^{2} y^{2} K}{\mathbb{E} \int_{B_{r_{2}}} y^{2} K \mathrm{~d} x} \mathrm{~d} t \\
\leq & 2 T\|a\|_{B_{r_{4}}}^{2}+8 \max |\nabla \varphi|^{2} \exp \left(-\frac{r_{3}-r_{2}}{4 \lambda}\right) \int_{T-2 \varepsilon}^{T} \frac{\mathbb{E} \int_{G \backslash B_{r_{3}}}|\nabla y|^{2} \mathrm{~d} x}{\mathbb{E} \int_{B_{r_{2}}} y^{2} \mathrm{~d} x} \mathrm{~d} t \\
& +2 \max |\Delta \varphi|^{2} \exp \left(-\frac{r_{3}-r_{2}}{4 \lambda}\right) \int_{T-2 \varepsilon}^{T} \frac{\mathbb{E} \int_{G \backslash B_{r_{3}}} y^{2} \mathrm{~d} x}{\mathbb{E} \int_{B_{r_{2}}} y^{2} \mathrm{~d} x} \mathrm{~d} t .
\end{aligned}
$$

Note that $r_{3}>r_{2}$, then for fixed $y$, we proved the claim that the term involving $F$ is uniformly bounded with respect to $\lambda \in[0,1]$.

From the definition of $H(\cdot)$, we see that

$$
\begin{aligned}
\frac{H(T-2 \varepsilon)}{H(T-\varepsilon)} & =\frac{\mathbb{E} \int_{G} \Phi^{2}(x, T-2 \varepsilon) K(x, T-2 \varepsilon) \mathrm{d} x}{\mathbb{E} \int_{G} \Phi^{2}(x, T-\varepsilon) K(x, T-\varepsilon) \mathrm{d} x} \\
& \leq\left(\frac{\lambda+\varepsilon}{\lambda+2 \varepsilon}\right)^{\frac{n}{2}} \frac{\mathbb{E} \int_{G} \Phi^{2}(x, T-2 \varepsilon) \vartheta\left(\lambda+2 \varepsilon, x, x_{0}\right) \mathrm{d} x}{\mathbb{E} \int_{G} \Phi^{2}(x, T-\varepsilon) \vartheta\left(\lambda+\varepsilon, x, x_{0}\right) \mathrm{d} x} .
\end{aligned}
$$

Denote the right hand side of the above inequality by $\mathcal{E}(\lambda)$. Then for fixed $\varepsilon$ and $\Phi$, it is clear that $\mathcal{E}(\lambda)$ is continuous in $(0,1]$ with respect to $\lambda$. Then, we know that $\mathcal{E}(\lambda)$ is uniformly bounded in $[0,1]$. So is $\mathcal{A}(\lambda)$.

Now returning to (3.4), noticing that $\lambda N(T) \leq \mathcal{A}(\lambda)$, we arrive at the following inequality:

$$
\left[1-\frac{8 \lambda}{r^{2}}\left(\mathcal{A}(\lambda)+\frac{n}{2}\right)\right] \mathbb{E} \int_{G}\left|x-x_{0}\right|^{2} \Phi^{2}(x, T) \vartheta\left(\lambda, x, x_{0}\right) \mathrm{d} x \leq 8 \lambda\left(\mathcal{A}(\lambda)+\frac{n}{2}\right) \mathbb{E} \int_{B_{r}} \Phi^{2}(x, T) \vartheta\left(\lambda, x, x_{0}\right) \mathrm{d} x .
$$

Since $\mathcal{A}(\cdot)$ is uniformly bounded in $(0,1]$, we can choose a $\lambda_{1} \in(0,1]$ such that

$$
\left[1-\frac{8 \lambda_{1}}{r^{2}}\left(\mathcal{A}\left(\lambda_{1}\right)+\frac{n}{2}\right)\right] \geq \frac{1}{2}
$$


This, together with (3.9), implies that

$$
\mathbb{E} \int_{G}\left|x-x_{0}\right|^{2} \Phi^{2}(x, T) \vartheta\left(\lambda_{1}, x, x_{0}\right) \mathrm{d} x \leq r^{2} \mathbb{E} \int_{B_{r}} \Phi^{2}(x, T) \vartheta\left(\lambda_{1}, x, x_{0}\right) \mathrm{d} x .
$$

Returning to the definition of $\Phi=\varphi y$ and the special properties of $\varphi$ and $r=r_{1}$, we arrive at that

$$
\mathbb{E} \int_{B_{r_{2}}}\left|x-x_{0}\right|^{2} y^{2}(x, T) \vartheta\left(\lambda_{1}, x, x_{0}\right) \mathrm{d} x \leq r_{1}^{2} \mathbb{E} \int_{B_{r_{1}}} y^{2}(x, T) \vartheta\left(\lambda_{1}, x, x_{0}\right) \mathrm{d} x .
$$

From (3.10), we know that we can use the data in a small ball to estimate that in a larger one. It follows that if $y(x, T)$ vanishes in $B_{r_{1}}, P$-a.s., then so does in $B_{r_{2}}$.

Next, we show that if $y$, which is the solution of (1.1), vanishes in a given small ball, it may vanishes everywhere in $G$. We prove this in the following manner.

For brevity, we use $B$ in place of $B_{r_{1}}$ in (3.10). For any ball $\widetilde{B}$ contained in $G$, we can construct two sequences of balls $\left\{S_{i}\right\}_{i=1}^{m}$ and $\left\{\widetilde{S}_{i}\right\}_{i=1}^{m-1}$ containing in $G$ for some finite natural number $m$, such that

$$
\left\{\begin{array}{l}
B \subset S_{1}, B \text { and } S_{1} \text { have the same center, } \\
\widetilde{S}_{i} \subset \subset S_{i} \cap S_{i+1}, \widetilde{S}_{i} \text { and } S_{i+1} \text { have the same center, } i=1,2, \ldots, m-1, \\
S_{m} \subset \widetilde{B}, S_{m} \text { and } \widetilde{B} \text { have the same center. }
\end{array}\right.
$$

By (3.10), if $y(\cdot, T)=0$ in $B$, so does it in $S_{1}$. Then by the selection of $\left\{S_{i}\right\}_{i=1}^{m}$ and $\left\{\widetilde{S}_{i}\right\}_{i=1}^{m-1}, y(\cdot, T)=0$ in $\widetilde{S}_{1}$ follows. So $y=0$ in $S_{2}, \ldots$, and so on. By induction, we conclude that if $y(\cdot, T)=0$ in $S_{i}$, so does it in $\widetilde{S}_{i} \subset \subset S_{i} \cap S_{i+1}, i=1,2, \ldots, m-1$. So $y(\cdot, T)=0$ in $S_{i+1}$ follows. we conclude that $y=0$ in $S_{m}$. At last, noticing that $S_{m} \subset \widetilde{B}$. Then use the same argument to get (3.10), we can conclude $y(\cdot, T)=0$ in $\widetilde{B}$.

Following the arbitrariness of $\widetilde{B}$, we conclude that if $y$, the solution of (1.1), vanishes $P$-a.s. in a small ball containing in $G$ at some fixed time $T$, so it vanishes $P$-a.s in $G$ at time $T$.

Next, if $y=0$ on $\partial G \times(0, T)$, then according to the backward uniqueness of stochastic heat equations (see Lem. 2.3), we know $y=0$ in $G \times(0, T), P$-a.s. Recalling also that $B_{r_{1}}=B_{r} \subset G_{0}$, we complete the proof.

\section{Proof of Theorem 1.6}

In this section, we give the quantitative unique continuation for the solution of equation (1.1) subject to Condition 1.4. For convenience, we adopt some notations.

For $K$ defined as in (2.1), we introduce that for $t \in[0, T]$,

$$
\left\{\begin{array}{l}
\widetilde{H}(t)=\mathbb{E} \int_{G}|y(x, t)|^{2} K(x, t) \mathrm{d} x \\
\widetilde{D}(t)=\mathbb{E} \int_{G}|\nabla y(x, t)|^{2} K(x, t) \mathrm{d} x \\
\widetilde{N}(t)=\frac{2 \widetilde{D}(t)}{\widetilde{H}(t)} \text { provided that } \widetilde{H}(t) \neq 0 .
\end{array}\right.
$$

Similar to the process of establishing Lemmas 2.1 and 2.2, we have the following two results.

Lemma 4.1. For $\widetilde{H}$ defined in (4.1), it holds that

$$
\widetilde{H}^{\prime}(t)=-2 \widetilde{D}(t)+2 \mathbb{E} \int_{G} a y^{2} K \mathrm{~d} x+\mathbb{E} \int_{G} b^{2} y^{2} K \mathrm{~d} x .
$$

Proof. The process is exactly following that of proving Lemma 2.1 . 
Lemma 4.2. Assume that $\widetilde{H} \neq 0$. For each $T>0$ and $0 \leq s<t \leq T$, it follows that

$$
\widetilde{N}(t)-\tilde{N}(s) \leq \int_{s}^{t}\left(\frac{1}{T-\tau+\lambda}+\|b\|_{G}^{2}\right) \widetilde{N}(\tau) \mathrm{d} \tau+\int_{s}^{t}\left(\|a\|_{G}^{2}+2\|b\|_{G}^{2}\right) \mathrm{d} \tau .
$$

Proof. The process is mimicking that for Lemma 2.2, so we omit some concrete calculations. The thing we should put more attention to is how to use the convex condition to deal with the boundary term appearing in the proof.

First, we can arrive at that

$$
\begin{aligned}
\widetilde{H}(t)-\widetilde{H}(s)= & 2 \mathbb{E} \int_{s}^{t} \int_{G} y\left(\Delta y+\frac{1}{2} a y-\frac{x-x_{0}}{2(T-t+\lambda)} \cdot \nabla y\right) K \mathrm{~d} x \mathrm{~d} \tau+\mathbb{E} \int_{s}^{t} \int_{G} a y^{2} K \mathrm{~d} x \mathrm{~d} \tau \\
& +\mathbb{E} \int_{s}^{t} \int_{G} b^{2} y^{2} K \mathrm{~d} x \mathrm{~d} \tau .
\end{aligned}
$$

Denote

$$
\mathcal{B}=\nabla \cdot\left(|\nabla y|^{2} \nabla K\right)-2 \nabla \cdot(\nabla y(\nabla y \cdot \nabla K))
$$

We have that We have that

$$
\begin{aligned}
\widetilde{D}(t)-\widetilde{D}(s)= & -2 \mathbb{E} \int_{s}^{t} \int_{G}\left[\Delta y+\frac{1}{2} a y-\frac{x-x_{0}}{2(T-t+\lambda)} \cdot \nabla y\right]^{2} K \mathrm{~d} x \mathrm{~d} \tau \\
& +\frac{1}{2} \mathbb{E} \int_{s}^{t} \int_{G} a^{2} y^{2} K \mathrm{~d} x \mathrm{~d} \tau-\mathbb{E} \int_{s}^{t} \int_{G} \mathcal{B} \mathrm{d} x \mathrm{~d} t \\
& +\frac{1}{T-t+\lambda} \int_{G}|\nabla y|^{2} K \mathrm{~d} x \mathrm{~d} t+\int_{G}|\nabla(b y)|^{2} K \mathrm{~d} x \mathrm{~d} t .
\end{aligned}
$$

We also have that

$$
\widetilde{D}(t)=-\mathbb{E} \int_{G} y\left[\Delta y+\frac{1}{2} a y-\frac{x-x_{0}}{2(T-t+\lambda)} \cdot \nabla y\right] K \mathrm{~d} x+\frac{1}{2} \mathbb{E} \int_{G} a y^{2} K \mathrm{~d} x .
$$

According to (4.4)-(4.7), we can find that for any $0 \leq s<t \leq T$,

$$
\begin{aligned}
\widetilde{N}(t)-\widetilde{N}(s)= & -\int_{s}^{t} \frac{4}{\widetilde{H}} \mathbb{E} \int_{G}\left[\Delta y+\frac{1}{2} a y-\frac{x-x_{0}}{2(T-\tau+\lambda)} \cdot \nabla y\right]^{2} K \mathrm{~d} x \mathrm{~d} \tau \\
& -\int_{s}^{t} \frac{2}{\widetilde{H}} \mathbb{E} \int_{G} \mathcal{B} \mathrm{d} x \mathrm{~d} \tau+\int_{s}^{t} \frac{1}{T-\tau+\lambda} \frac{2 \widetilde{D}}{\widetilde{H}} \mathrm{~d} \tau+\int_{s}^{t} \frac{2}{\widetilde{H}} \mathbb{E} \int_{G}|\nabla(b y)|^{2} \mathrm{~d} x \mathrm{~d} \tau \\
& +\int_{s}^{t} \frac{4}{\widetilde{H}^{2}}\left[\mathbb{E} \int_{G} y\left(\Delta y+\frac{1}{2} a y-\frac{x-x_{0}}{2(T-\tau+\lambda)} \cdot \nabla y \mathrm{~d} t\right) K \mathrm{~d} x\right]^{2} \mathrm{~d} \tau \\
& -\int_{s}^{t} \frac{1}{\widetilde{H}^{2}}\left(\mathbb{E} \int_{G} a y^{2} K \mathrm{~d} x\right)^{2} \mathrm{~d} \tau-\int_{s}^{t} \frac{2 \widetilde{D}}{\widetilde{H}^{2}} \mathbb{E} \int_{G} b^{2} y^{2} K \mathrm{~d} x \mathrm{~d} t+\int_{s}^{t} \frac{1}{\widetilde{H}} \mathbb{E} \int_{G} a^{2} y^{2} K \mathrm{~d} x \mathrm{~d} \tau .
\end{aligned}
$$


For $G$ being convex, then $\left(x-x_{0}\right) \cdot \nu \geq 0$ for each $x \in \partial G$ with $\nu$ the out unit normal vector at $x$. Also, noting that $y$, which solves the equation (1.1), vanishes on the boundary $\partial G$, one finds that

$$
\begin{aligned}
\int_{s}^{t} \mathbb{E} \int_{G} \mathcal{B} \mathrm{d} x \mathrm{~d} \tau & =\int_{s}^{t} \mathbb{E} \int_{G}\left[\nabla \cdot\left(|\nabla y|^{2} \nabla K\right)-2 \nabla \cdot(\nabla y(\nabla y \cdot \nabla K))\right] \mathrm{d} x \mathrm{~d} \tau \\
& =\int_{s}^{t} \mathbb{E} \int_{\partial G}\left[\left(|\nabla y|^{2} \nabla K\right)-2(\nabla y(\nabla y \cdot \nabla K))\right] \cdot \nu \mathrm{d} S \mathrm{~d} \tau \\
& =\int_{s}^{t} \mathbb{E} \int_{\partial G}\left[-|\nabla y|^{2} \frac{\left(x-x_{0}\right) \cdot \nu}{2(T-\tau+\lambda)}+2 \frac{x-x_{0}}{2(T-\tau+\lambda)} \cdot \nabla y(\nabla y \cdot \nu)\right] K \mathrm{~d} S \mathrm{~d} \tau \\
& =\int_{s}^{t} \mathbb{E} \int_{\partial G}\left[-|\nabla y|^{2} \frac{\left(x-x_{0}\right) \cdot \nu}{2(T-\tau+\lambda)}+2|\nabla y|^{2} \frac{\left(x-x_{0}\right) \cdot \nu}{2(T-\tau+\lambda)}\right] K \mathrm{~d} S \mathrm{~d} \tau \\
& \geq 0,
\end{aligned}
$$

where we use the symbol d $S$ to represent the boundary measure.

Combining (4.8) with (4.9), it follows that

$$
\begin{aligned}
\tilde{N}(t)-\widetilde{N}(s) & \leq \int_{s}^{t} \frac{1}{T-\tau+\lambda} \frac{2 \widetilde{D}}{\widetilde{H}} \mathrm{~d} \tau+\int_{s}^{t} \frac{2}{\widetilde{H}} \mathbb{E} \int_{G}|\nabla(b y)|^{2} \mathrm{~d} x \mathrm{~d} \tau+\int_{s}^{t} \frac{1}{\widetilde{H}} \mathbb{E} \int_{G} a^{2} y^{2} K \mathrm{~d} x \mathrm{~d} \tau \\
& \leq \int_{s}^{t}\left(\frac{1}{T-\tau+\lambda}+\|b\|_{G}^{2}\right) \widetilde{N}(\tau) \mathrm{d} \tau+\int_{s}^{t}\left(\|a\|_{G}^{2}+2\|b\|_{G}^{2}\right) \mathrm{d} \tau .
\end{aligned}
$$

Remark 4.3. For Theorem 1.6, we assume the convexity of the domain $G$. We use this assumption to deal with the boundary terms as shown in (4.9). How to get rid of this condition has its independent interest.

We are now in a position to give the Proof of Theorem 1.6.

Proof of Theorem 1.6. For simplicity, we always assume that $T_{0}=T$ in this section. If $y(\cdot, T)=0$ in $G, P$-a.s., then the inequality $(1.7)$ holds. Now we only consider the case that $y(\cdot, T) \neq 0$ in $G, P$-a.s. In this case, due to Lemma 2.3 , we know that $y(\cdot, t) \neq 0$ in $G, P$-a.s.

Note that $B_{r} \subset G_{0} \subset \subset G$ is the ball centered at $x_{0}$ with radius $r$. As in the proof for Theorem 1.2, we begin with calculating

$$
\int_{G}\left|\nabla\left[y(x, T) \vartheta\left(2 \lambda, x, x_{0}\right)\right]\right|^{2} \mathrm{~d} x \geq 0 .
$$

Via some straightforward calculations, similar to the process of getting (3.4), we arrive at that

$$
\left[1-\frac{8 \lambda}{r^{2}}\left(\lambda \widetilde{N}(T)+\frac{n}{2}\right)\right] \mathbb{E} \int_{G}\left|x-x_{0}\right|^{2} y^{2}(x, T) \vartheta\left(\lambda, x, x_{0}\right) \mathrm{d} x \leq 8 \lambda\left(\lambda \widetilde{N}(T)+\frac{n}{2}\right) \mathbb{E} \int_{B_{r}} y^{2}(x, T) \vartheta\left(\lambda, x, x_{0}\right) \mathrm{d} x .
$$

In what follows, we turn to estimate $\lambda \widetilde{N}(T)$.

From the estimate for $\widetilde{N}$ in Lemma 4.2 and Gronwall's inequality, it follows that

$$
\begin{aligned}
\lambda \tilde{N}(T) \leq & \exp \left((T-t)\|b\|_{G}^{2}\right)(T-t+\lambda) \tilde{N}(t) \\
& +\exp \left((T-t)\|b\|_{G}^{2}\right)(T-t+\lambda)(T-t)\left(\|a\|_{G}^{2}+2\|b\|_{G}^{2}\right) \\
\leq & \exp \left(T\|b\|_{G}^{2}\right)(T+\lambda) \widetilde{N}(t)+\exp \left(T\|b\|_{G}^{2}\right)(T+\lambda) T\left(\|a\|_{G}^{2}+2\|b\|_{G}^{2}\right) .
\end{aligned}
$$


Integrating (4.12) with time variable $t$ over $\left[0, \frac{T}{2}\right]$, we have that

$$
\frac{T}{2} \lambda \widetilde{N}(T) \leq(T+\lambda) \exp \left(T\|b\|_{G}^{2}\right) \int_{0}^{\frac{T}{2}} \widetilde{N}(\tau) \mathrm{d} \tau+(T+\lambda) \frac{T^{2}}{2} \exp \left(T\|b\|_{G}^{2}\right)\left(\|a\|_{G}^{2}+2\|b\|_{G}^{2}\right) .
$$

Turning to the result of Lemma 4.1, say (4.2), it is easy to show that

$$
\int_{0}^{\frac{T}{2}} \widetilde{N}(\tau) \mathrm{d} \tau \leq \ln \frac{\widetilde{H}(0)}{\widetilde{H}\left(\frac{T}{2}\right)}+T\left(\|a\|_{G}+2\|b\|_{G}^{2}\right) .
$$

Thus, we obtain that

$$
\lambda \widetilde{N}(T) \leq \frac{2(T+\lambda)}{T} \exp \left(T\|b\|_{G}^{2}\right)\left[\ln \frac{\widetilde{H}(0)}{\widetilde{H}\left(\frac{T}{2}\right)}+\frac{T}{2}\left(2\|a\|_{G}+T\|a\|_{G}^{2}+(1+2 T)\|b\|_{G}^{2}\right)\right] .
$$

Denote $m=\max _{x \in \bar{G}}\left|x-x_{0}\right|^{2}$. By some elementary calculations, we find that

$$
\begin{aligned}
\frac{\widetilde{H}(0)}{\widetilde{H}\left(\frac{T}{2}\right)} & =\left(\frac{\frac{T}{2}+\lambda}{T+\lambda}\right)^{\frac{n}{2}} \frac{\mathbb{E} \int_{G} y^{2}(x, 0) \vartheta\left(T+\lambda, x, x_{0}\right) \mathrm{d} x}{\mathbb{E} \int_{G} y^{2}\left(x, \frac{T}{2}\right) \vartheta\left(T / 2+\lambda, x, x_{0}\right) \mathrm{d} x} \\
& \leq \exp \left(\frac{m}{4\left(\lambda+\frac{T}{2}\right)}\right) \frac{\mathbb{E} \int_{G} y^{2}(x, 0) \mathrm{d} x}{\mathbb{E} \int_{G} y^{2}\left(x, \frac{T}{2}\right) \mathrm{d} x} \\
& \leq \exp \left(\frac{m}{2 T}\right) \frac{\mathbb{E} \int_{G} y^{2}(x, 0) \mathrm{d} x}{\mathbb{E} \int_{G} y^{2}\left(x, \frac{T}{2}\right) \mathrm{d} x} .
\end{aligned}
$$

Note that $y$ solves equation (1.1). By Itô's formula, we have that

$$
\begin{aligned}
\mathbb{E} \int_{G} y^{2}(x, t) \mathrm{d} x & =\mathbb{E} \int_{G} y^{2}(x, s) \mathrm{d} x+\mathbb{E} \int_{s}^{t} \int_{G}\left(2 a y^{2}+b^{2} y^{2}-2|\nabla y|^{2}\right) \mathrm{d} x \mathrm{~d} \tau \\
& \leq \mathbb{E} \int_{G} y^{2}(x, s) \mathrm{d} x+\mathbb{E} \int_{s}^{t} \int_{G}\left(2 a y^{2}+b^{2} y^{2}\right) \mathrm{d} x \mathrm{~d} \tau \\
& \leq \mathbb{E} \int_{G} y^{2}(x, s) \mathrm{d} x+\left(2\|a\|_{G}+\|b\|_{G}^{2}\right) \mathbb{E} \int_{s}^{t} \int_{G} y^{2} \mathrm{~d} x \mathrm{~d} \tau
\end{aligned}
$$

holds for $0 \leq s \leq t \leq T$. Due to Gronwall's inequality, it follows that

$$
\mathbb{E} \int_{G} y^{2}(x, T) \mathrm{d} x \leq \exp \left(T\left(\|a\|_{G}+\frac{\|b\|_{G}^{2}}{2}\right)\right) \mathbb{E} \int_{G} y^{2}\left(x, \frac{T}{2}\right) \mathrm{d} x .
$$

According to (4.14) and (4.15), we obtain that

$$
\frac{\widetilde{H}(0)}{\widetilde{H}\left(\frac{T}{2}\right)} \leq \exp \left(\frac{m}{2 T}+T\left(\|a\|_{G}+\frac{\|b\|_{G}^{2}}{2}\right)\right) \frac{\mathbb{E} \int_{G} y^{2}(x, 0) \mathrm{d} x}{\mathbb{E} \int_{G} y^{2}(x, T) \mathrm{d} x} .
$$

Making use of (4.13) and (4.16), it is easy to show that

$$
\begin{aligned}
\lambda \widetilde{N}(T) \leq & (T+\lambda) \exp \left(T\|b\|_{G}^{2}\right)\left[\frac{2}{T} \ln \frac{\mathbb{E} \int_{G} y^{2}(x, 0) \mathrm{d} x}{\mathbb{E} \int_{G} y^{2}(x, T) \mathrm{d} x}+\frac{m}{T^{2}}\right. \\
& \left.+\left(4\|a\|_{G}+T\|a\|_{G}^{2}+2(1+T)\|b\|_{G}^{2}\right)\right]
\end{aligned}
$$


Fix $\lambda \in(0,1]$. Denote

$$
\begin{aligned}
\mathcal{D}= & (T+1) \exp \left(T\|b\|_{G}^{2}\right)\left[\frac{2}{T} \ln \frac{\mathbb{E} \int_{G} y^{2}(x, 0) \mathrm{d} x}{\mathbb{E} \int_{G} y^{2}(x, T) \mathrm{d} x}\right. \\
& \left.+\frac{m}{T^{2}}+\left(4\|a\|_{G}+T\|a\|_{G}^{2}+2(1+T)\|b\|_{G}^{2}\right)\right]+\frac{n}{2} .
\end{aligned}
$$

We find that

$$
\lambda \widetilde{N}(T)+\frac{n}{2} \leq \mathcal{D}
$$

This together with (4.11) implies

$$
\left(1-\frac{8 \lambda}{r^{2}} \mathcal{D}\right) \mathbb{E} \int_{G}\left|x-x_{0}\right|^{2} y^{2}(x, T) \vartheta\left(\lambda, x, x_{0}\right) \mathrm{d} x \leq 8 \lambda \mathcal{D} \mathbb{E} \int_{B_{r}} y^{2}(x, T) \vartheta\left(\lambda, x, x_{0}\right) \mathrm{d} x .
$$

Letting $\widetilde{\lambda}=\frac{r^{2}}{16 \mathcal{D}}$, we arrive at that

$$
\mathbb{E} \int_{G}\left|x-x_{0}\right|^{2} y^{2}(x, T) \vartheta\left(\widetilde{\lambda}, x, x_{0}\right) \mathrm{d} x \leq r^{2} \mathbb{E} \int_{B_{r}} y^{2}(x, T) \vartheta\left(\widetilde{\lambda}, x, x_{0}\right) \mathrm{d} x .
$$

Due to (4.18), an elementary computation gives that

$$
\begin{aligned}
\mathbb{E} \int_{G} y^{2}(x, T) \vartheta\left(\widetilde{\lambda}, x, x_{0}\right) \mathrm{d} x & =\mathbb{E} \int_{G \backslash B_{r}} y^{2}(x, T) \vartheta\left(\widetilde{\lambda}, x, x_{0}\right) \mathrm{d} x+\mathbb{E} \int_{B_{r}} y^{2}(x, T) \vartheta\left(\widetilde{\lambda}, x, x_{0}\right) \mathrm{d} x \\
& \leq \frac{1}{r^{2}} \mathbb{E} \int_{G}\left|x-x_{0}\right|^{2} y^{2}(x, T) \vartheta\left(\widetilde{\lambda}, x, x_{0}\right) \mathrm{d} x+\mathbb{E} \int_{B_{r}} y^{2}(x, T) \vartheta\left(\widetilde{\lambda}, x, x_{0}\right) \mathrm{d} x \\
& \leq 2 \mathbb{E} \int_{B_{r}} y^{2}(x, T) \vartheta\left(\widetilde{\lambda}, x, x_{0}\right) \mathrm{d} x
\end{aligned}
$$

Noting the definition of $m$ and the choice of $\widetilde{\lambda}$, we follows from (4.19) that

$$
\mathbb{E} \int_{G} y^{2}(x, T) \mathrm{d} x \leq 2 \exp \left(\frac{4 m \mathcal{D}}{r^{2}}\right) \mathbb{E} \int_{B_{r}} y^{2}(x, T) \mathrm{d} x .
$$

Put

$$
\begin{aligned}
\mathcal{J} & =\mathcal{D}-\frac{2(T+1)}{T} \exp \left(T\|b\|_{G}^{2}\right) \ln \frac{\mathbb{E} \int_{G} y^{2}(x, 0) \mathrm{d} x}{\mathbb{E} \int_{G} y^{2}(x, T) \mathrm{d} x} \\
& =(T+1) \exp \left(T\|b\|_{G}^{2}\right)\left[\frac{m}{T^{2}}+\left(4\|a\|_{G}+T\|a\|_{G}^{2}+2(1+T)\|b\|_{G}^{2}\right)\right]+\frac{n}{2} .
\end{aligned}
$$

Plugging $\mathcal{J}$ into (4.20), one has that

$$
\mathbb{E} \int_{G} y^{2}(x, T) \mathrm{d} x \leq 2 \exp \left(\frac{4 m \mathcal{J}}{r^{2}}\right)\left(\frac{\mathbb{E} \int_{G} y^{2}(x, 0) \mathrm{d} x}{\mathbb{E} \int_{G} y^{2}(x, T) \mathrm{d} x}\right)^{\theta} \mathbb{E} \int_{B_{r}} y^{2}(x, T) \mathrm{d} x,
$$

where $\theta=\frac{8 m(T+1) \exp \left(T\|b\|_{G}^{2}\right)}{r^{2} T}$.

After some elementary calculations, one finds that

$$
\mathbb{E} \int_{G} y^{2}(x, T) \mathrm{d} x \leq 2^{\delta} \exp (\beta)\left(\mathbb{E} \int_{G} y^{2}(x, 0) \mathrm{d} x\right)^{1-\delta}\left(\mathbb{E} \int_{B_{r}} y^{2}(x, T) \mathrm{d} x\right)^{\delta} .
$$


In (4.21), the simple notations $\delta$ and $\beta$ are given as follows:

$$
\left\{\begin{array}{l}
\delta=\frac{r^{2} T}{r^{2} T+8 m(T+1) \exp \left(T\|b\|_{G}^{2}\right)}, \\
\beta=\frac{4 m T \mathcal{J}}{r^{2} T+8 m(T+1) \exp \left(T\|b\|_{G}^{2}\right)} .
\end{array}\right.
$$

Noting that $B_{r} \subset G_{0}$, we then complete the Proof of Theorem 1.6.

\section{Proof of Theorem 1.10}

This section is devoted to the Proof of Theorem 1.10. To begin with, we first introduce the following lemma, whose proof can be found in reference [21].

Lemma 5.1. Let $E \subset(0, T)$ be a measurable set of positive measure. Let $t_{0}$ be a density point of $E$. Then for each $z>1$, there exists an $t_{1} \in\left(t_{0}, T\right)$ such that the sequence $\left\{t_{m}\right\}_{m=1}^{\infty}$, given by

$$
t_{m+1}=t_{0}+\frac{1}{z^{m}}\left(t_{1}-t_{0}\right)
$$

satisfies

$$
t_{m}-t_{m+1} \leq 3\left|E \cap\left(t_{m+1}, t_{m}\right)\right| .
$$

Proof of Theorem 1.10. Let $t_{0}$ be a density point of $E$. Let $\left\{t_{m}\right\}_{m=1}^{\infty}$ be a sequence provided by Lemma 5.1. From (4.21), it follows that for any $t \in[0, T]$,

$$
\mathbb{E} \int_{G} y^{2}(x, t) \mathrm{d} x \leq \frac{2}{\varepsilon^{\gamma}} \exp (\Theta) \mathbb{E} \int_{B_{r}} y^{2}(x, t) \mathrm{d} x+\varepsilon \mathbb{E} \int_{G} y^{2}(x, 0) \mathrm{d} x,
$$

where

$$
\Theta=\max _{t \in[0, T]} \frac{t \mathcal{J}}{2(t+1) \exp \left(t\|b\|_{G}^{2}\right)}, \quad \gamma=\max _{t \in[0, T]} \frac{8 m(t+1) \exp \left(t\|b\|_{G}^{2}\right)}{r^{2} t+8 m(t+1) \exp \left(t\|b\|_{G}^{2}\right)} .
$$

For the solution to (1.1), by standard argument, it is easy to verify the following energy estimate:

$$
\mathbb{E} \int_{G} y^{2}(x, t) \mathrm{d} x \leq \exp (C(a, b) t) \mathbb{E} \int_{G} y^{2}(x, 0) \mathrm{d} x,
$$

where

$$
C(a, b, t)=\left(2\|a\|_{G}^{2}+\|b\|_{G}^{2}\right) t .
$$

For $t \in\left(t_{m+1}, t_{m}\right]$, combining (5.3) and (5.4), one finds that

$$
\begin{aligned}
\exp (-C(a, b, T)) \mathbb{E} \int_{G} y^{2}\left(x, t_{m}\right) \mathrm{d} x & \leq \mathbb{E} \int_{G} y^{2}(x, t) \mathrm{d} x \\
& \leq \frac{2}{\varepsilon^{\gamma}} \exp (\Theta) \mathbb{E} \int_{B_{r}} y^{2}(x, t) \mathrm{d} x+\varepsilon \mathbb{E} \int_{G} y^{2}\left(x, t_{m+1}\right) \mathrm{d} x .
\end{aligned}
$$

As a direct result of this inequality, we get that

$$
\varepsilon^{\gamma} \exp (-C(a, b, T)) \mathbb{E} \int_{G} y^{2}\left(x, t_{m}\right) \mathrm{d} x-\varepsilon^{\gamma+1} \mathbb{E} \int_{G} y^{2}\left(x, t_{m+1}\right) \mathrm{d} x \leq 2 \exp (\Theta) \mathbb{E} \int_{B_{r}} y^{2}(x, t) \mathrm{d} x .
$$


Integrating over $E \cap\left(t_{m+1}, t_{m}\right)$ shows that

$$
\begin{aligned}
\left|E \cap\left(t_{m+1}, t_{m}\right)\right|\left[\varepsilon^{\gamma} \exp (-C(a, b, T)) \mathbb{E} \int_{G} y^{2}\left(x, t_{m}\right) \mathrm{d} x-\varepsilon^{\gamma+1} \mathbb{E} \int_{G} y^{2}\left(x, t_{m+1}\right) \mathrm{d} x\right] & \leq 2 \exp (\Theta) \mathbb{E} \int_{E \cap\left(t_{m+1}, t_{m}\right)} \int_{B_{r}} y^{2}(x, t) \mathrm{d} x .
\end{aligned}
$$

Letting $\alpha_{m}=\varepsilon^{\gamma}\left|E \cap\left(t_{m+1}, t_{m}\right)\right|, \sigma_{m}=\varepsilon^{\gamma+1}\left|E \cap\left(t_{m+1}, t_{m}\right)\right|$, then it is clear that

$$
\alpha_{m} \exp (-C(a, b, T)) \mathbb{E} \int_{G} y^{2}\left(x, t_{m}\right) \mathrm{d} x-\sigma_{m} \mathbb{E} \int_{G} y^{2}\left(x, t_{m+1}\right) \mathrm{d} x \leq 2 \exp (\Theta) \mathbb{E} \int_{E \cap\left(t_{m+1}, t_{m}\right)} \int_{B_{r}} y^{2}(x, t) \mathrm{d} x .
$$

We choose a sequence of $\left\{\varepsilon_{m}\right\}_{m=1}^{\infty}$ in the following way: Let $\sigma_{m}=\alpha_{m+1} \exp (-C(a, b, T))$ such that

$$
\frac{\varepsilon_{m}^{\gamma+1}}{\varepsilon_{m+1}^{\gamma}} \exp (C(a, b, T))=\frac{\left|E \cap\left(t_{m+2}, t_{m+1}\right)\right|}{\left|E \cap\left(t_{m+1}, t_{m}\right)\right|}
$$

By means of the properties of $\left\{t_{m}\right\}_{m=1}^{\infty}$ mentioned in Lemma 5.1, this implies that for any $m \in \mathbb{N}$,

$$
\begin{aligned}
\varepsilon_{m+1}^{\gamma} & =\varepsilon_{m}^{\gamma+1} \exp (C(a, b, T)) \frac{\left|E \cap\left(t_{m+1}, t_{m}\right)\right|}{\left|E \cap\left(t_{m+2}, t_{m+1}\right)\right|} \\
& \leq \varepsilon_{m}^{\gamma+1} \exp (C(a, b, T)) \frac{3\left|\left(t_{m+1}, t_{m}\right)\right|}{\left|\left(t_{m+2}, t_{m+1}\right)\right|} \\
& \leq 3 z \varepsilon_{m}^{\gamma+1} \exp (C(a, b, T)) .
\end{aligned}
$$

Let us choose $\varepsilon_{1}=\frac{1}{3 z \exp (C(a, b, T))}$. Then from (5.6), we see that $\varepsilon_{2}^{\gamma} \leq \varepsilon_{1}^{\gamma}$. Hence, we know that $\varepsilon_{2} \leq$ $\frac{1}{3 z \exp (C(a, b, T))}$. This, together with (5.6), implies that $\varepsilon_{3} \leq \frac{1}{3 z \exp (C(a, b))}$. By induction, we find that

$$
\varepsilon_{m} \leq \frac{1}{3 z \exp (C(a, b, T))} \text { for any } m \in \mathbb{N} .
$$

Consequently, following (5), one finds that

$$
\begin{aligned}
& \sum_{m=1}^{n}\left(\alpha_{m} \exp (-C(a, b, T)) \mathbb{E} \int_{G} y^{2}\left(x, t_{m}\right) \mathrm{d} x-\sigma_{m} \mathbb{E} \int_{G} y^{2}\left(x, t_{m+1}\right) \mathrm{d} x\right) \\
& \quad \leq 2 \exp (\Theta) \sum_{m=1}^{n} \mathbb{E} \int_{E \cap\left(t_{m+1}, t_{m}\right)} \int_{B_{r}} y^{2}(x, t) \mathrm{d} x,
\end{aligned}
$$

which implies that

$$
\alpha_{1} \exp (-C(a, b, T)) \mathbb{E} \int_{G} y^{2}\left(x, t_{1}\right) \mathrm{d} x-\sigma_{n} \mathbb{E} \int_{G} y^{2}\left(x, t_{n+1}\right) \mathrm{d} x \leq 2 \exp (\Theta) \mathbb{E} \int_{E} \int_{B_{r}} y^{2}(x, t) \mathrm{d} x .
$$

It is noted that

$$
\lim _{n \rightarrow \infty} \sigma_{n}=\lim _{n \rightarrow \infty} \varepsilon_{n}^{\gamma+1}\left|E \cap\left(t_{n+1}, t_{n}\right)\right|=0
$$

following the construction of $\left\{t_{n}\right\}_{n=1}^{\infty}$ and that $0<\varepsilon_{n}<\frac{1}{3 z \exp (C(a, b, T))}$. Therefore, in light of (5.7), we immediately arrive at

$$
\mathbb{E} \int_{G} y^{2}\left(x, t_{1}\right) \mathrm{d} x \leq 2 \alpha_{1}^{-1} \exp (C(a, b, T)) \exp (\Theta) \mathbb{E} \int_{E} \int_{B_{r}} y^{2}(x, t) \mathrm{d} x .
$$

This inequality together with the energy estimate (5.4) shows that

$$
\mathbb{E} \int_{G} y^{2}(x, T) \mathrm{d} x \leq 2 \alpha_{1}^{-1} \exp (2 C(a, b, T)) \exp (\Theta) \mathbb{E} \int_{E} \int_{B_{r}} y^{2}(x, t) \mathrm{d} x .
$$

Letting $C=2 \alpha_{1}^{-1} \exp (2 C(a, b, T)) \exp (\Theta)$ and noting that $B_{r} \subset G_{0}$, we complete the desired result. 


\section{Proof of Theorems 1.9 And 1.12}

Proof of Theorem 1.9. Due to the linearity of (1.9), it suffices to show that its attainable set $A_{T}$ at time $T$ with final datum $z(T)=0$ is dense in $L^{2}(G)$. Let us prove this by the contradiction argument.

If $A_{T}$ is not dense in $L^{2}(G)$, then there exists an $\eta \in L^{2}(G)$ such that $\eta \neq 0$ and

$$
\int_{G} z(0) \eta \mathrm{d} x=0 \text { for any } z(0) \in A_{T} \text {. }
$$

Let us consider the following equation

$$
\begin{cases}\mathrm{d} \tilde{y}-\Delta \tilde{y} \mathrm{~d} t=-a_{1} \tilde{y} \mathrm{~d} t-b_{1} \tilde{y} \mathrm{~d} B(t) & \text { in } G \times(0, T), \\ \tilde{y}=0 & \text { on } \partial G \times(0, T), \\ \tilde{y}(0)=\eta & \text { in } G .\end{cases}
$$

It is clear that the solution $\tilde{y}$ to $(6.1)$ belongs to $L_{\mathcal{W}}^{2}\left(\Omega ; C\left([0, T] ; L^{2}(G)\right)\right) \cap L_{\mathcal{W}}^{2}\left(0, T ; H_{0}^{1}(G)\right)$.

From Itô's formula, we find that

$$
\begin{aligned}
\mathbb{E} \int_{G} \tilde{y}(T) z(T) \mathrm{d} x-\mathbb{E} \int_{G} \tilde{y}_{0} z(0) \mathrm{d} x= & \mathbb{E} \int_{0}^{T} \int_{G}\left[\tilde{y}\left(-\Delta z+a_{1} z+b_{1} Z+h+\chi_{E_{1}} \chi_{G_{0}} f\right)\right. \\
& \left.+z\left(\Delta y-a_{1} \tilde{y}\right)-b_{1} \tilde{y} Z\right] \mathrm{d} x \mathrm{~d} t \\
= & \mathbb{E} \int_{0}^{T} \int_{G} \tilde{y} h \mathrm{~d} x \mathrm{~d} t+\mathbb{E} \int_{0}^{T} \int_{G} \tilde{y} \chi_{E_{1}} \chi_{G_{0}} f \mathrm{~d} x \mathrm{~d} t .
\end{aligned}
$$

Let $z(T)=0$, we obtain that

$$
0=\mathbb{E} \int_{G} z(0) \eta \mathrm{d} x=\mathbb{E} \int_{0}^{T} \int_{G} \tilde{y} h \mathrm{~d} x \mathrm{~d} t+\mathbb{E} \int_{0}^{T} \int_{G} \tilde{y} \chi_{E_{1}} \chi_{G_{0}} f \mathrm{~d} x \mathrm{~d} t .
$$

Hence,

$$
\mathbb{E} \int_{0}^{T} \int_{G} \tilde{y} h \mathrm{~d} x \mathrm{~d} t+\mathbb{E} \int_{0}^{T} \int_{G} \tilde{y} \chi_{E_{1}} \chi_{G_{0}} f \mathrm{~d} x \mathrm{~d} t=0
$$

for any $f \in L_{\mathcal{W}}^{2}\left(0, T ; L^{2}(G)\right)$. Therefore we get

$$
\tilde{y}=0 \text { in } G_{0} \times E_{1}, P \text {-a.s. }
$$

Then, from Theorem 1.2, we arrive at $\eta=0$, a contradiction.

Proof of Theorem 1.12. Consider the following equation:

$$
\begin{cases}\mathrm{d} \hat{y}-\Delta \hat{y} \mathrm{~d} t=-a_{1} \hat{y} \mathrm{~d} t-b_{1} \hat{y} \mathrm{~d} B(t) & \text { in } G \times(0, T), \\ \hat{y}=0 & \text { on } \partial G \times(0, T) .\end{cases}
$$

We introduce a linear subspace of $L_{\mathcal{W}}^{2}\left(0, T ; L^{2}\left(G_{0}\right)\right)$ :

$$
\mathcal{X} \triangleq\left\{\left.\hat{y}\right|_{G_{0} \times E_{1}}: \hat{y} \text { solves equation (6.4) with some initial datum } \hat{y}_{0} \in L^{2}(G)\right\},
$$

and define a linear functional $\mathcal{L}$ on $\mathcal{X}$ as follows:

$$
\mathcal{L}\left(\left.\hat{y}\right|_{G_{0} \times E_{1}}\right)=-\mathbb{E} \int_{G} y(T) z_{T} \mathrm{~d} x .
$$


By Theorem 1.10, we see that

$$
\begin{aligned}
\left|\mathcal{L}\left(\left.\hat{y}\right|_{G_{0} \times E_{1}}\right)\right| & \leq\|y(T)\|_{L^{2}\left(\Omega, \mathcal{F}_{T}, P ; L^{2}(G)\right)}\left\|z_{T}\right\|_{L^{2}\left(\Omega, \mathcal{F}_{T}, P ; L^{2}(G)\right)} \\
& \leq \mathcal{C}\left\|z_{T}\right\|_{L^{2}\left(\Omega, \mathcal{F}_{T}, P ; L^{2}(G)\right)}\left(\mathbb{E} \int_{0}^{T} \int_{G_{0}}\left|\hat{\chi}_{E_{1}} y\right|^{2} \mathrm{~d} x \mathrm{~d} t\right)^{\frac{1}{2}} .
\end{aligned}
$$

Therefore, $\mathcal{L}$ is a bounded linear functional on $\mathcal{X}$. By Hahn-Banach Theorem, $\mathcal{L}$ can be extended to a bounded linear functional with the same norm on $L_{\mathcal{F}}^{2}\left(0, T ; L^{2}\left(G_{0}\right)\right)$. For simplicity, we use the same notation for this extension. Now, Riesz Representation Theorem allows us to find a random field $f \in L_{\mathcal{F}}^{2}\left(0, T ; L^{2}\left(G_{0}\right)\right)$ so that

$$
\mathbb{E} \int_{0}^{T} \int_{G} \hat{y} \chi_{E_{1}} \chi_{G_{0}} f \mathrm{~d} x \mathrm{~d} t=\mathbb{E} \int_{G} y(T) z_{T} \mathrm{~d} x .
$$

We claim that this $f$ is the control we need. In fact, for any $z_{T} \in L_{\mathcal{W}_{T}}^{2}\left(\Omega ; L^{2}(G)\right)$, for the solution $\hat{y}$ of equation (6.4) and the solution $(z, Z)$ of equation (1.9), by Itô's formula, we get that

$$
\begin{aligned}
\mathbb{E} \int_{G} \hat{y}(T) z(T) \mathrm{d} x-\mathbb{E} \int_{G} \hat{y}_{0} z(0) \mathrm{d} x & =\mathbb{E} \int_{0}^{T} \int_{G}\left[\hat{y}\left(-\Delta z+a_{1} z+b_{1} Z+\chi_{E_{1}} \chi_{G_{0}} f\right)+z\left(\Delta y-a_{1} \hat{y}\right)-b_{1} \hat{y} Z\right] \mathrm{d} x \mathrm{~d} t \\
& =\mathbb{E} \int_{0}^{T} \int_{G} \hat{y} \chi_{E_{1}} \chi_{G_{0}} f \mathrm{~d} x \mathrm{~d} t
\end{aligned}
$$

Combining (6.5) and (6.6), we find that

$$
\mathbb{E} \int_{G} \hat{y}_{0} z(0) \mathrm{d} x=0
$$

Since $\hat{y}_{0}$ can be chosen arbitrarily, we know that $z(0)=0$ in $G$.

Acknowledgements. Qi Lü would like to thank the Laboratoire Jacques-Louis Lions (LJLL) at Université Pierre et Marie Curie for its hospitality. Part of this work was carried out while he was visiting the LJLL.

\section{REFERENCES}

[1] J.P. Dauer, N.I. Mahmudov and M.M. Matar, Approximate controllability of backward stochastic evolution equations in Hilbert spaces. J. Math. Anal. Appl. 323 (2006) 42-56.

[2] L. de Teresa, Approximate controllability of a semilinear heat equation in $\mathbf{R}^{N}$. SIAM J. Control Optim. 36 (1998) $2128-2147$.

[3] L. Escauriaza, Carleman inequalities and the heat operator. Duke Math. J. 104 (2000) 113-127.

[4] L. Escauriaza and L. Vega, Carleman inequalities and the heat operator II. Indiana Univ. Math. J. 50 (2001) $1149-1169$.

[5] C. Fabre, J.-P. Puel and E. Zuazua, Approximate controllability of the semilinear heat equation. Proc. Roy. Soc. Edinburgh Sect. A 125 (1995) 31-61.

[6] E. Fernández-Cara and S. Guerrero, Global Carleman inequalities for parabolic systems and applications to controllability. SIAM J. Control Optim. 45 (2006) 1399-1446.

[7] E. Fernández-Cara and E. Zuazua, The cost of approximate controllability for heat equations: the linear case. Adv. Differ. Equ. 5 (2000) 465-514.

[8] E. Fernández-Cara and E. Zuazua, Null and approximate controllability for weakly blowing up semilinear heat equations. Ann. Inst. Henri Poincaré Anal., Non Linéaire 17 (2000) 583-616.

[9] E. Fernández-Cara, M.J. Garrido-Atienza and J. Real, On the approximate controllability of a stochastic parabolic equation with a multiplicative noise. C. R. Acad. Sci. Paris Sér. I Math. 328 (1999) 675-680.

[10] A.V. Fursikov and O.Yu. Imanuvilov, Controllability of Evolution Equations. Vol. 34 of Lect. Notes Ser. Seoul National University, Research Institute of Mathematics, Global Analysis Research Center, Seoul (1996).

[11] J. Hadamard, Lectures on Cauchy's problem in linear partial differential equations. Dover Publications, New York (1953).

[12] L. Hörmander, The Analysis of Linear Partial differential operators. III. Pseudodifferential operators. Vol. 274 of Grundlehren der Mathematischen Wissenschaften [Fundamental Principles of Mathematical Sciences]. Springer-Verlag, Berlin (1985). 
[13] M.M. Lavrentév, V.G. Romanov and S.P. Shishat-skii, Ill-posed Problems of Mathematical Physics and Analysis, Translated from the Russian by J.R. Schulenberger. Vol. 64 of Trans. Math. Monogr. AMS, Providence, RI (1986).

[14] G. Lebeau and L. Robbiano, Contrôle exact de l'équation de la chaleur. Commun. Partial Differ. Equ. 20 (1995) 335-356.

[15] H. Li and Q. Lü, A quantitative boundary unique continuation for stochastic parabolic equations. J. Math. Anal. Appl. 402 (2013) 518-526.

[16] F. Lin, A uniqueness theorem for parabolic equations. Commun. Pure Appl. Math. 43 (1990) 127-136.

[17] Q. Lü, Some results on the controllability of forward stochastic heat equations with control on the drift. J. Funct. Anal. 260 (2011) 832-851.

[18] Q. Lü, Carleman estimate for stochastic parabolic equations and inverse stochastic parabolic problems. Inverse Probl. 28 (2012) 045008.

[19] N.I. Mahmudov, Approximate controllability of semilinear deterministic and stochastic evolution equations in abstract spaces. SIAM J. Control Optim. 42 (2003) 1604-1622.

[20] K.-D. Phung and G. Wang, Quantitative unique continuation for the semilinear heat equation in a convex domain. J. Funct. Anal. 259 (2010) 1230-1247.

[21] K.-D. Phung and G. Wang, An observability estimate for parabolic equations from a measurable set in time and its applications. J. Eur. Math. Soc. 15 (2013) 681-703.

[22] K.-D. Phung, L. Wang and C. Zhang, Bang-bang property for time optimal control of semilinear heat equation, Ann. Inst. Henri Poincaré, Anal. Non Linéaire 31 (2014) 477-499.

[23] C. Poon, Unique continuation for parabolic equations. Commun. Partial Differ. Equ. 21 (1996) 521-539.

[24] J. Real, Some results on controllability for stochastic heat and Stokes equations. C. R. Acad. Sci. Paris Sér. I Math. 322 (1996) 1197-1202.

[25] S. Tang and X. Zhang, Null controllability for forward and backward stochastic parabolic equations. SIAM J. Control Optim. 48 (2009) 2191-2216.

[26] S. Vessella, Carleman estimates, optimal three cylinder inequality, and unique continuation properties for solutions to parabolic equations. Commun. Partial Differ. Equ. 28 (2003) 637-676.

[27] M. Yamamoto, Carleman estimates for parabolic equations and applications. Inverse Probl. 25 (2009) 123013.

[28] X. Zhang, Unique continuation for stochastic parabolic equations. Differ. Integral Equ. 21 (2008) 81-93.

[29] X. Zhou, A duality analysis on stochastic partial differential equations. J. Funct. Anal. 103 (1992) 275-293.

[30] E. Zuazua, Controllability and observability of partial differential equations: some results and open problems. Vol. III of Handbook of differential equations: evolutionary equations. Elsevier/North-Holland, Amsterdam (2007).

[31] C. Zuily, Uniqueness and nonuniqueness in the Cauchy Problem. Birkhäuser Boston, Inc., Boston, MA (1983). 